\title{
The LIMD1 protein bridges an association between the prolyl hydroxylases and VHL to repress HIF-1 activity
}

\author{
Daniel E. Foxler ${ }^{1,8}$, Katherine S. Bridge ${ }^{1,8}$, Victoria James ${ }^{1,8}$, Thomas M. Webb ${ }^{2,8}$, Maureen Mee ${ }^{1}$, \\ Sybil C. K. Wong ${ }^{1}$, Yunfeng Feng ${ }^{3}$, Dumitru Constantin-Teodosiu ${ }^{1}$, Thorgunnur Eyfjord Petursdottir ${ }^{4}$, \\ Johannes Bjornsson $^{4}$, Sigurdur Ingvarsson ${ }^{5}$, Peter J. Ratcliffe ${ }^{6}$, Gregory D. Longmore ${ }^{3}$ and Tyson V. Sharp ${ }^{1,7,9}$
}

There are three prolyl hydroxylases (PHD1, 2 and 3) that regulate the hypoxia-inducible factors (HIFs), the master transcriptional regulators that respond to changes in intracellular $\mathrm{O}_{2}$ tension ${ }^{1,2}$. In high $\mathrm{O}_{2}$ tension (normoxia) the PHDs hydroxylate two conserved proline residues on HIF-1 $\alpha$, which leads to binding of the von Hippel-Lindau (VHL) tumour suppressor, the recognition component of a ubiquitin-ligase complex, initiating HIF-1 $\alpha$ ubiquitylation and degradation ${ }^{3-6}$. However, it is not known whether PHDs and VHL act separately to exert their enzymatic activities on HIF-1 $\alpha$ or as a multiprotein complex. Here we show that the tumour suppressor protein LIMD1 (LIM domain-containing protein) acts as a molecular scaffold, simultaneously binding the PHDs and VHL, thereby assembling a PHD-LIMD1-VHL protein complex and creating an enzymatic niche that enables efficient degradation of HIF-1 $\alpha$. Depletion of endogenous LIMD1 increases HIF-1 $\alpha$ levels and transcriptional activity in both normoxia and hypoxia. Conversely, LIMD1 expression downregulates HIF-1 transcriptional activity in a manner depending on PHD and 26S proteasome activities. LIMD1 family member proteins Ajuba and WTIP also bind to VHL and PHDs 1 and 3, indicating that these LIM domain-containing proteins represent a previously unrecognized group of hypoxic regulators.

LIMD1 is a tumour suppressor and member of the Ajuba family of LIM domain-containing proteins ${ }^{7,8}$. This family of proteins is characterized by three conserved carboxy-terminal LIM domains involved in protein-protein interactions arrayed in tandem in addition to a less well-characterized and divergent proline/serine-rich amino-terminal (pre-LIM) region ${ }^{9,10}$. A yeast two-hybrid screen identified PHD1 as a specific binding partner of LIMD1 (Supplementary Fig. S1a). As LIMD1 shares homology with Ajuba family member proteins Ajuba and WTIP, and within the LIM-domain region with the zyxin LIM domain-containing family members TRIP6, LPP and zyxin, we investigated if these also bound PHD enzymes. We carried out in vivo co-immunoprecipitation assays with Xpress-tagged family member proteins and PHD1, 2 and 3 and discovered differential binding of the PHDs to the family members (Fig. 1a). LIMD1 bound all three PHDs, whereas the closely related WTIP and Ajuba only bound PHD1 and 3. Out of the more distantly related zyxin family members, the only interaction detected was between TRIP6 and a modified higher-molecularweight form of PHD1 of unknown function (denoted by an arrow in Fig. 1a). Owing to the homology of the PHD family members and the demonstration that PHD2 is the critical oxygen sensor responsible for the regulation of HIF $\alpha$ (refs 11,12), we focused our attention on this hydroxylase together with LIMD1 (which bound all three PHDs). In a direct binding assay recombinant LIMD1 bound directly to glutathione $S$-transferase (GST)-PHD2 in vitro (Supplementary Fig. S1b-d).

On the basis of these findings we asked if LIMD1's tumoursuppressive function could, in part, be through formation of a PHD-LIMD1-VHL active complex regulating the HIF-1 $\alpha$ degradation pathway, and thus one or more of the many downstream HIF-target genes that have pro-angiogenic, pro-metastatic or other oncogenic functions ${ }^{13}$. To test this possibility, we first immunoprecipitated endogenous PHD2 and found endogenous LIMD1 to co-immunoprecipitate in addition to a higher-molecular-weight form of LIMD1 of unknown modification (most probably representing phosphorylated forms ${ }^{14}$, unpublished data; Fig. 1b). Immunoprecipitation of endogenous VHL from HEK293 cells resulted in the co-precipitation of elongin B, cullin 2, LIMD1 and PHD2, further supporting the presence of this complex in cells (Fig. 1c). Furthermore,

${ }^{1}$ School of Biomedical Sciences, University of Nottingham, Queen's Medical Centre, NG7 2UH, UK. ${ }^{2}$ BDU, BioPharm, Discovery R\&D, GSK, Gunnells Wood Road, Stevenage SG1 2NY, UK. ${ }^{3}$ Departments of Medicine and Cell Biology and Physiology, The BRIGHT Institute, Washington University, St Louis, Missouri 63110, USA.

${ }^{4}$ Department of Pathology, Landspitali University Hospital, Reykjavik, Iceland. Institute for Experimental Pathology and Faculty of Medicine, University of Iceland at Keldur, Reykjavik, Iceland. ${ }^{6}$ Henry Wellcome Building for Molecular Physiology, University of Oxford, Oxford OX3 7BN, UK. ${ }^{7}$ Present Address: Barts Cancer Institute, Queen Mary University of London, Charterhouse Square, London EC1M 6BQ, UK. ${ }^{8}$ These authors contributed equally to this work.

${ }^{9}$ Correspondence should be addressed to T.V.S. (e-mail: t.sharp@qmul.ac.uk) 
sucrose-gradient analysis of HEK293 cell extracts demonstrated that LIMD1 was present in the same fractions as PHD2, VHL, elongin B and cullin 2 (Supplementary Fig. S1e). These biochemical data supported the existence of a PHD2-LIMD1-VHL complex and also corroborate an earlier in vivo study, where PHD2 purified in a complex with an apparent relative molecular mass above $M_{\mathrm{r}} 300,000$ (300K) (ref. 15).

The members of the Ajuba family function as protein adapters, simultaneously binding multiple proteins to enable specific regulatory processes $^{10,16}$. To assess if this function was applicable to HIF-1 regulation we examined if those family members able to bind one or more of the PHDs were also able to bind VHL. In vivo co-immunoprecipitation revealed that both the p19 and p30 isoforms of VHL (refs 17,18) bound specifically to LIMD1, Ajuba and WTIP but not zyxin or LPP (Fig. 1d). To further validate the endogenous VHL and LIMD1 interaction we also identified this association in HeLa and U2OS cells (Supplementary Fig. S13f,g). These data therefore support the concept that LIMD1 is part of a complex that contains the VHL E3 ligase. Of note, we repeatedly observed multiple forms of LIMD1 and VHL in these endogenous co-immunoprecipitation studies (Fig. 1b,c and Supplementary Fig. S1f,g). These multiple co-precipitated forms of LIMD1 and VHL were specific, as neither control experiments that probed the immunoprecipitating antibodies alone nor experiments carried out in the VHL-deficient RCC4 cell line showed any nonspecific cross-reactivity (Supplementary Fig. S1i-k). The multiple forms of VHL match those observed in the sucrose-gradient analysis and have previously been reported ${ }^{19}$. The higher-molecular-weight forms of LIMD1 appear only on concentration of the complex through immunoprecipitation and thus may be specifically modified as a result of complex formation. Such an association between LIMD1 and VHL would predict that they form a functional E3 ligase complex. Consistent with this hypothesis, we detected increased HIF-1 $\alpha$ ubiquitylation following LIMD1 overexpression (Fig. 1e). A PHD2/VHL non-binding mutant ( $\Delta 186-220 \Delta$ LIM2, see below) was unable to induce ubiquitylation (Fig. 1e).

We next sought to determine which region of LIMD1 mediates its association with PHD2 and VHL. In vivo co-immunoprecipitation studies with the pre-LIM region of LIMD1 (LIMD1 $\Delta 472-676$ ) and LIM-domain region (LIMD1 $\Delta 1-467$; Fig. 1f) revealed PHD2 bound to the pre-LIM region (Fig. 1g) and VHL bound to the LIM-domain region (Fig. 1h). These data together with the endogenous co-immunoprecipitation and sucrose-gradient data suggest that LIMD1 may be able to simultaneously bind PHD2 and VHL and therefore may enhance the co-operative function of both proteins by increasing their local concentration, enabling more efficient sequential modification and degradation of HIF-1 $\alpha$. As VHL does not associate directly with PHDs, this hypothesis predicts that the presence of LIMD1 would enable VHL to associate in a complex also containing PHD2 in vivo. In ectopic expression studies, immunoprecipitation of VHL in the absence of transfected plasmids encoding LIMD1 resulted in only very low levels of associated PHD2 (Fig. 1i); however, when plasmids encoding LIMD1 were co-transfected this resulted in significantly increased co-precipitation of PHD2 with VHL (Fig. 1i). Furthermore, immunoprecipitation of transfected plasmids encoding LIMD1 in the presence of PHD2 and VHL resulted in the association of both proteins with LIMD1 and importantly neither impaired the other's ability to bind LIMD1 (Supplementary Fig. S1l,m), as would be expected if both proteins interacted with distinct regions of LIMD1 (Fig. 1g,h) and if LIMD1 acts as an adapter for both proteins. LIMD1 did not bind full-length HIF-1 $\alpha$ or its separate sub-domains as determined by in vivo co-immunoprecipitation (Supplementary Fig. S2a,b).

We next tested if overexpression of LIMD1 affected HIF-1 transcriptional activity. Ectopic expression of LIMD1 with HIF-1 $\alpha$ resulted in significantly reduced hypoxic response element (HRE)driven luciferase reporter activity (Fig. 2a). This was specific for LIMD1, as LPP (PHD/VHL non-binder) did not cause any significant repression (Fig. 2b). Furthermore, HRE transcription in the presence and absence of co-expressed PHD2 indicated that the negative effect of LIMD1 on HIF- $1 \alpha$ transcriptional activity was additive with exogenous PHD2 (Fig. 2b). Western blot analysis of the reporter lysates revealed that LIMD1 caused a decrease in HIF- $1 \alpha$ levels (Supplementary Fig. S2c)

To corroborate the LIMD1 ectopic-expression studies we carried out the converse analysis in cell lines specifically depleted for LIMD1 using lentiviral-driven short hairpin RNA (shRNA) technology and, as a control for potential off-target effects of LIMD1 depletion, in the absence or presence of RNA interference (RNAi)-resistant LIMD1 (rrLIMD1) expressed from the same lentiviral vector ${ }^{20}$ (Fig. 2c). Depletion of LIMD1 either in normoxia $\left(20 \% \mathrm{O}_{2}\right)$ or hypoxia $\left(1 \% \mathrm{O}_{2}\right)$ resulted in increased HIF activity, which was restored (or in fact significantly repressed) by concurrent expression of RNAi-resistant LIMD1 (Fig. 2c). As predicted by the action of LIMD1 on HIF-1 $\alpha$ degradation (Supplementary Fig. S2c), short interfering RNA (siRNA)-targeted depletion of endogenous LIMD1 increased endogenous HIF-1 $\alpha$ protein levels in normoxia (Fig. 2d) and was comparable to that observed following siRNA-targeted depletion of PHD2 (Fig. 2e).

When LIMD1-depleted cells were exposed to hypoxia we observed a greatly exaggerated increase in HIF- $1 \alpha$ protein levels (Fig. 2f). This result would at first seem paradoxical owing to the inability of VHL to recognize and ubiquitylate unmodified HIF- $1 \alpha$ at $1 \%$ oxygen. However, it is now clear that in both acute and chronically hypoxic cells PHD2 activity is still present, acting to degrade HIF- $1 \alpha$ (refs 21-23). In addition, VHL can engage HIF-1 $\alpha$ in hypoxia and promote VHL-mediated HIF- $1 \alpha$ ubiquitylation and degradation ${ }^{24}$. We therefore reasoned that the PHD2-LIMD1-VHL complex may also be functional in hypoxia, potentially as part of the adaptive response to chronic hypoxia, and that depletion of LIMD1 in these conditions would disrupt the complex, leading to further HIF- $1 \alpha$ stabilization. To test this possibility, we recapitulated the experiments of ref. 22 with siRNA-targeted depletion of LIMD1 in acute and chronic hypoxia $\left(4-72 \mathrm{~h}\right.$ at $1 \% \mathrm{O}_{2}$; Fig. $\left.2 \mathrm{~g}\right)$. These experiments revealed that LIMD1 depletion, similarly to PHD2 depletion, caused a significant stabilization of HIF- $1 \alpha$ protein in both acute and chronic hypoxia (in addition to normoxia) and inhibited adaptation to chronic hypoxia (Fig. 2g; compare HIF-1 $\alpha$ expression in control lanes with siRNA depletion of LIMD1 and PHD2 over time in hypoxia). Furthermore, HIF-1 $\alpha$ degradation was inhibited following re-oxygenation of hypoxic cells depleted of LIMD1 (Supplementary Fig. S3a). These results therefore suggest that in hypoxia a significant proportion of cellular HIF- $1 \alpha$ continues to undergo LIMD1-dependent degradation; of this only a small proportion is stabilized under hypoxic conditions. The two distinct molecular-weight forms of HIF- $1 \alpha$ consistently observed in the above 

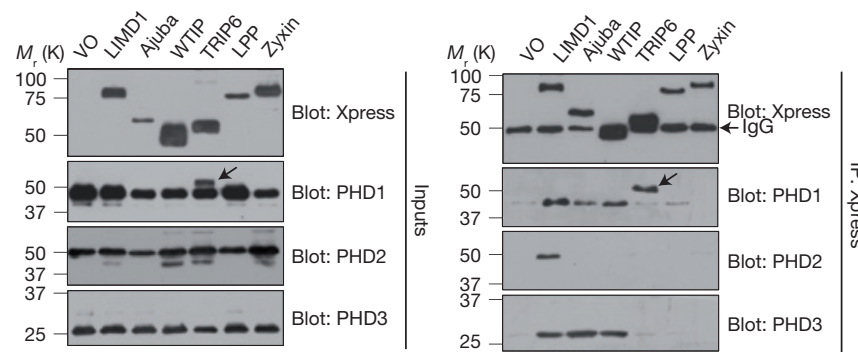

d
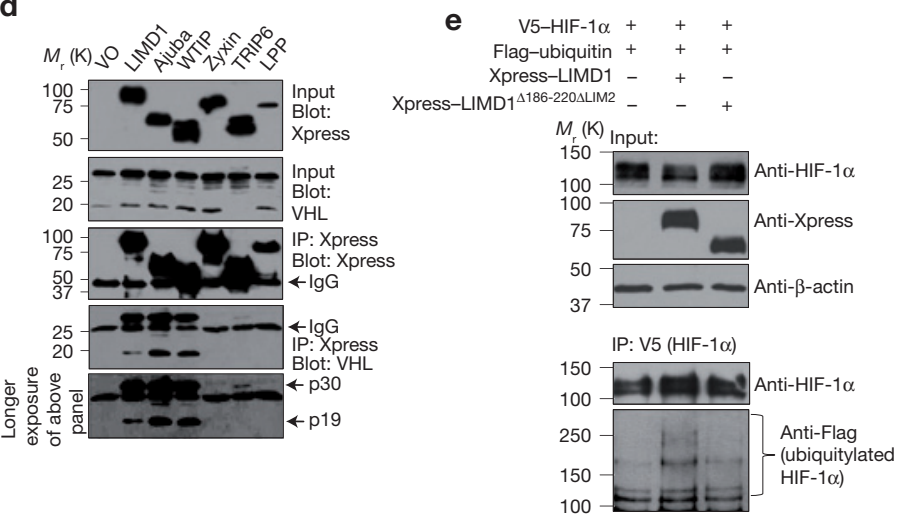

b

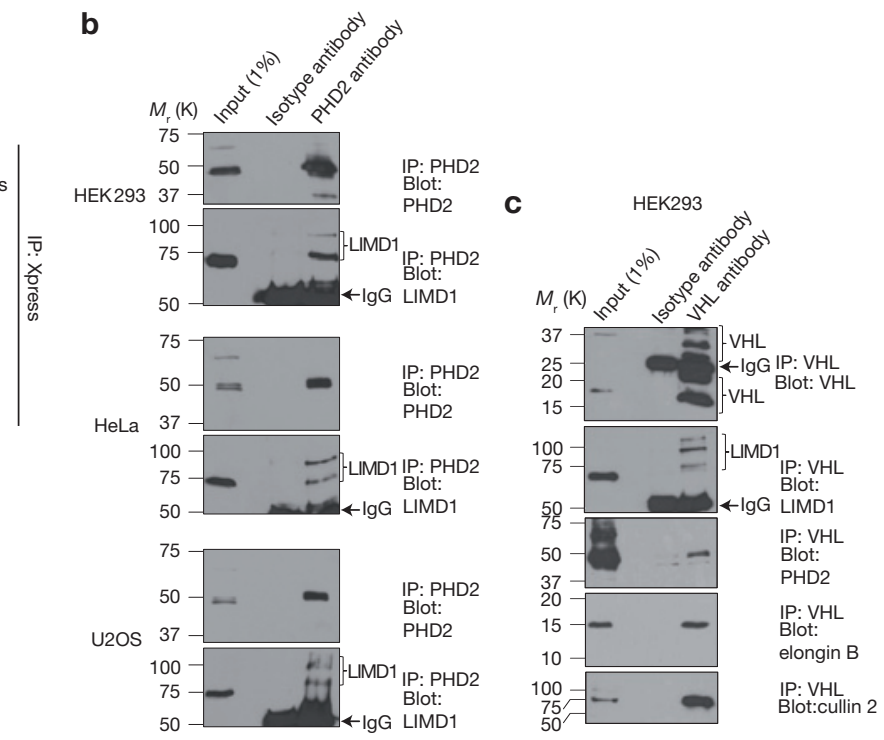

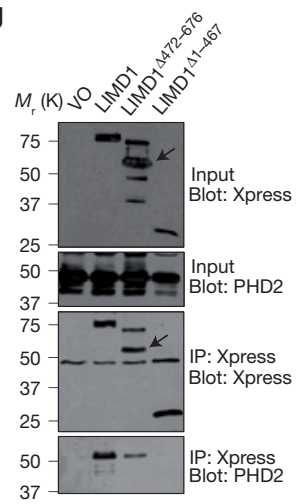

h

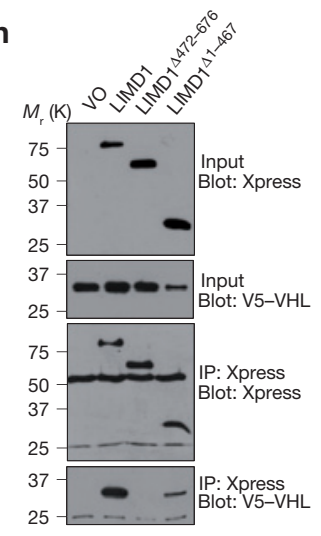

i

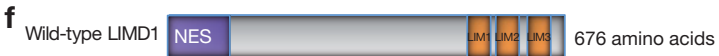

LIMD1 $^{\triangle 472-676} \square$

$\mathrm{LIMD1}^{\Delta 1-467}$

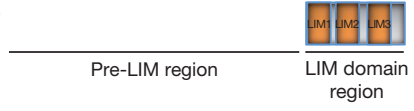

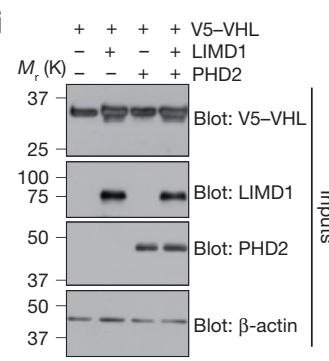

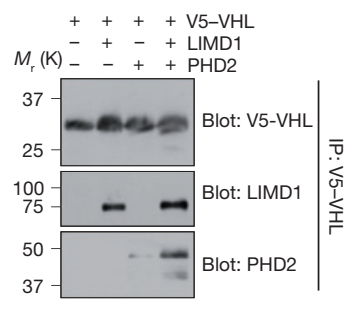

Figure 1 The Ajuba/zyxin family members interact differentially with PHD1-3 and VHL. (a) Plasmids encoding Xpress-tagged Ajuba/zyxin family members were co-transfected with untagged pcDNA3-PHD1, 2 or 3 into U2OS cells. $1 \%$ inputs loaded indicate protein levels before addition of the immunoprecipitating (IP) Xpress antibody. The family members interact differentially with the PHDs, with LIMD1 being the only family member to bind all three and the only member to bind PHD2. (b) Endogenous PHD2 was immunoprecipitated with PHD2 antibody ( $5 \mu \mathrm{g}$ ) from HEK293, HeLa or U2OS cell lysates. Endogenous LIMD1 co-immunoprecipitated with endogenous PHD2 in all the cell lysates assayed. (c) Endogenous VHL was immunoprecipitated from HEK293 cell lysate with VHL antibody ( $5 \mu \mathrm{g})$. VHL co-immunoprecipitated endogenous LIMD1 and the VCB complex proteins elongin $B$ and cullin 2. (d) The indicated family members were co-transfected with VHL into U2OS cells. Both the p30 and p19 VHL isoforms were expressed. The family members were immunoprecipitated with Xpress antibody. LIMD1, Ajuba and WTIP all bound to the p19 and p30 forms of VHL. TRIP6 only bound the p30 form (longer-exposure panel) and zyxin and LPP did not bind to either. (e) Ubiquitylation assay of V5-HIF-1 $\alpha$. Lysates from HEK293 cells transfected with V5-HIF-1 $\alpha$ vector were mixed with

assays (arrows, Fig. 2e-g and Supplementary Fig. S3a) are due to HIF- $1 \alpha$ phosphorylation ${ }^{25-28}$ as determined by $\lambda$-protein phosphatase treatment (Supplementary Fig. S3b).

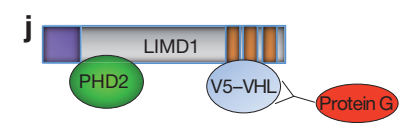

lysates from cells co-transfected with Flag-ubiquitin vector and incubated at $37^{\circ} \mathrm{C}$. Immunoprecipitation was carried out with V5 antibody and western blot with Flag antibody. (f) Schematic representation of LIMD1 depicting the three C-terminal LIM domains and a non-conserved pre-LIM region. NES, nuclear export signal. The interface within LIMD1 responsible for binding with PHD2 and VHL was examined using Xpress-tagged wild-type LIMD1, pre-LIM ( $\Delta 472-676)$ and LIM domain-only mutants $(\Delta 1-467)$. $(\mathbf{g}, \mathbf{h})$ The Xpress constructs were immunoprecipitated with Xpress antibody. Arrows indicate specific bands in lane 3 (LIMD1 $\triangle 472-676$ ). PHD2 binds to LIMD1 through the pre-LIM domain (g) whereas VHL interacts through the LIM domain (h). (i) V5-tagged VHL was immunoprecipitated using a V5 antibody, following co-transfection with plasmids encoding LIMD1 or PHD2 individually and in combination. LIMD1 co-immunoprecipitated with $\mathrm{VHL}$ regardless of ectopically expressed PHD2. Co-immunoprecipitation of PHD2 was significantly enhanced with overexpressed LIMD1, suggesting that LIMD1 simultaneously binds VHL and PHD2 into a protein complex. (j) Illustration of PHD2-LIMD1-VHL complex and co-immunoprecipitation approach used. In all panels, VO or - indicates vector only/empty vector was added as control.

In normoxia, degradation of HIF- $1 \alpha$ is a result of hydroxylation and ubiquitylation within the oxygen-dependent degradation domain (ODD) of HIF-1 $\alpha$ (refs 6,29). As expression of LIMD1 decreases 

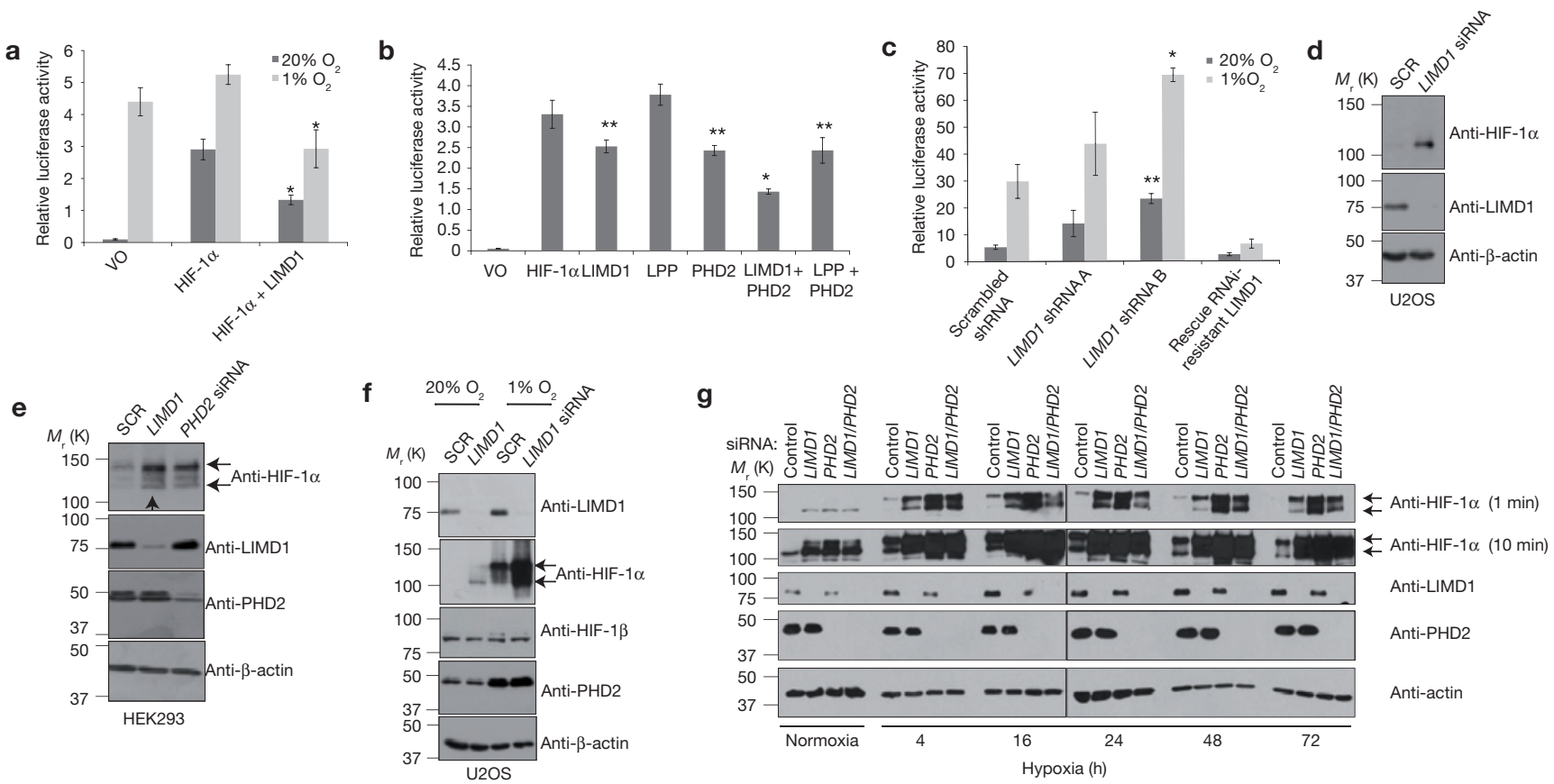

Figure 2 LIMD1 is a negative regulator of HIF-1 $\alpha$ levels and transcription activity. (a) Ectopic co-expression of LIMD1 with HIF-1 $\alpha$ represses synthetic pGL3-HRE-luc activity in normoxia and hypoxia when compared with expression of HIF-1 $\alpha$ alone. HRE-luc was normalized to a thymidine kinase (TK)-driven Renilla luciferase. Data are mean \pm s.d., $n=3$ independent experiments, ${ }^{*} P<0.005$. (b) Plasmids encoding LIMD1 or LPP were co-transfected plus or minus $1 \mathrm{ng}$ of PHD2. LIMD1 and PHD2 caused HRE repression in an additive manner. LPP did not cause HRE repression and when co-transfected with plasmids encoding PHD2 the level of repression was similar to that for PHD2 expression alone. Data are mean \pm s.d., $n=3$ independent experiments, ${ }^{*} P<0.005$, ${ }^{* *} P<0.05$. (c) Lentiviral-driven shRNAs $(\mathrm{A}$ and $\mathrm{B})$ were used to deplete endogenous LIMD1 and rescue with an RNAi-resistant LIMD1 in U2OS cells as confirmed by western blot. LIMD1 shRNA A and B represent

HIF-1 $\alpha$ levels (Supplementary Fig. S2c), we asked if increased LIMD1 expression would have a specific negative effect on stability of the ODD domain of HIF- $1 \alpha$ alone. Expression of LIMD1 resulted in reduced levels of ODD, but not of the amino (amino acids 30-389)- or carboxy (amino acids 630-826)-terminal domains (Fig. 3a). In contrast, TRIP6, which does not bind PHD2 (Fig. 1a), did not induce ODD degradation (Fig. 3a,c).

We next used the shRNA lentiviral-infected cell lines to test the converse prediction with respect to ODD protein levels and LIMD1 expression. Depletion of LIMD1 resulted in an increase in ODD expression (arrowhead) that is reduced with re-expression of rrLIMD1 (Fig. 3b). Family members Ajuba and WTIP, which bind both PHD and VHL, were also able to induce ODD degradation (Fig. 3c).

The dependence on hydroxylase and proteasomal activities for LIMD1-mediated regulation of ODD levels was examined next. In the presence of DMOG (a competitive inhibitor of 2-oxoglutarate (2-OG)-dependent oxygenases) or MG132 (a 26S proteasome inhibitor), LIMD1, Ajuba, WTIP and PHD2 were unable to induce a reduction in ODD levels, confirming a requirement for both enzymatic activities (Fig. 3d,e).

A further prediction of our proposed PHD2-LIMD1-VHL complex would be that PHD2/VHL-non-binding LIMD1 mutants would be unable to induce degradation of ODD or HIF, deregulate HIF activity
shRNAs that target different sites within the LIMD1 mRNA 3' untranslated region ( $3^{\prime}$ UTR) and $5^{\prime}$ UTR respectively. Rescue experiments were carried out on the shRNA B-depleted background. shRNA-mediated LIMD1 depletion results in enhancement of synthetic pGL3-HRE-luc activity in both normoxia and hypoxia. This is reversed by re-expression of an RNAi-resistant LIMD1. Data are mean \pm s.d., $n=3$ independent experiments, ${ }^{*} P<0.005,{ }^{* *} P<0.05$. (d,e) siRNA (80 nM)-mediated depletion of LIMD1 in U2OS (d) and HEK293 (e) cells causes an increase in HIF-1 $\alpha$ protein levels in normoxia comparable to that caused by siRNA depletion of PHD2. SCR, scrambled. (f) siRNA-mediated depletion of LIMD1 ( $80 \mathrm{nM})$ in U2OS cells also caused an increase in HIF-1 $\alpha$ protein levels in hypoxia and had no effect on HIF-1 $\beta$ protein levels. (g) siRNA-mediated depletion of LIMD1, PHD2 or both caused an increase in HIF-1 $\alpha$ protein levels in normoxia and up to $72 \mathrm{~h}$ hypoxia.

in reporter assays or may even act in a dominant inhibitory manner. To create non-binding mutants in the context of full-length LIMD1 the internal binding sites within LIMD1 were mapped for PHD2 ( $\Delta 186-260)$ and VHL ( $\triangle$ LIM2; Supplementary Fig. S4a,b, respectively).

As predicted, the double $\mathrm{PHD} 2 / \mathrm{VHL}$ non-binding mutant ( $\Delta 186-260-\Delta$ LIM2) did not induce ODD degradation when overexpressed in the presence of endogenous LIMD1 (Fig. 3f), indicating the requirement of associated PHD hydroxylase and/or VHL ubiquitin-ligase activities for LIMD1 to regulate this pathway. In an endogenous LIMD1-depleted background the capability of rrLIMD1 (expressed at levels comparable to endogenous LIMD1) to degrade ODD was lost on rescue with $\mathrm{rr} \Delta 186-260-\Delta$ LIM2 or rr $\Delta$ LIM2 mutants (Fig. 3g). In contrast, rescue with $\mathrm{rr} \Delta 186-260$ alone was able to influence ODD degradation. These results suggest that the VHL association with LIMD1 is pivotal for LIMD1-induced HIF degradation, and could also suggest a LIMD1-independent PHD modification of HIF-1 $\alpha$. These ODD results were mirrored when the same cell lines were analysed for HRE activity in normoxia (Fig. 3h). Interestingly, when the same assay was carried out under hypoxia the $\operatorname{rr} \Delta \mathrm{LIM} 2$ mutants resulted in a dominant negative effect on HRE-luc transcription (Fig. 3 compare $\mathrm{h}$ and i). This indicated (1) that in hypoxia engaging VHL is critical or (2) that the ability to bind and sequester PHD2 away from VHL may further stabilize 
a

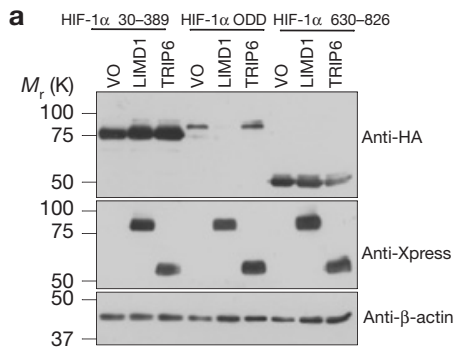

b
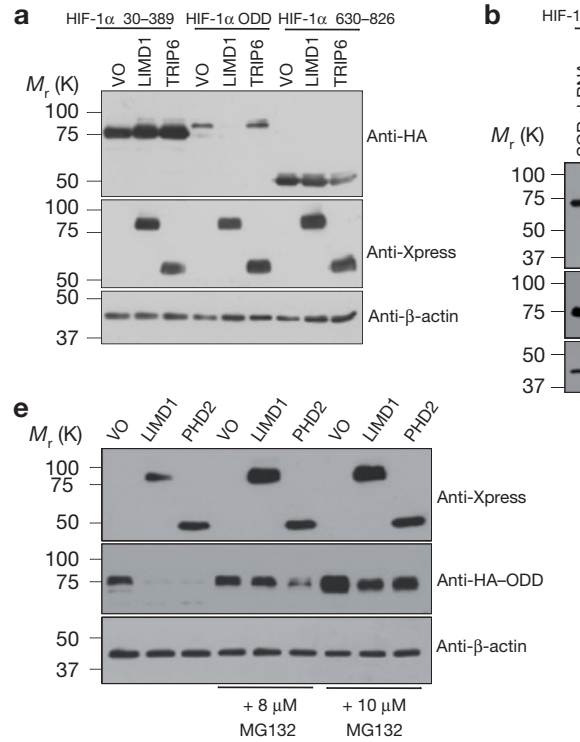

h

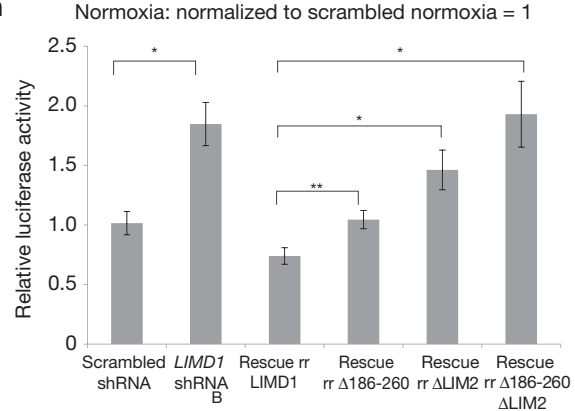

i

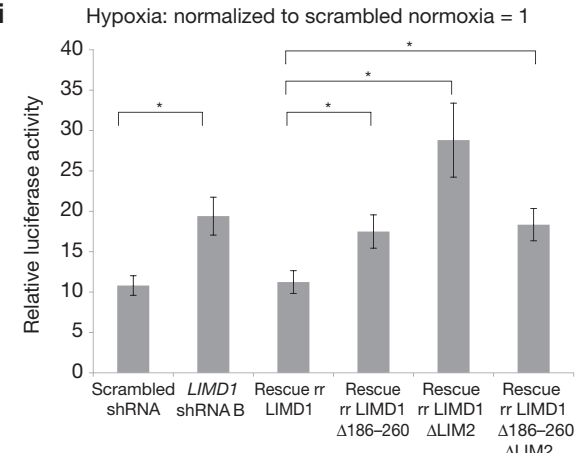

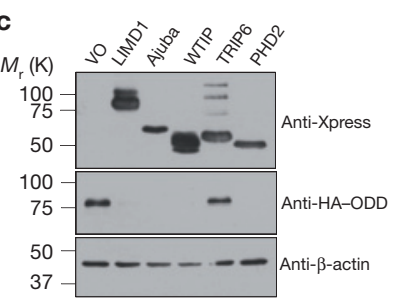

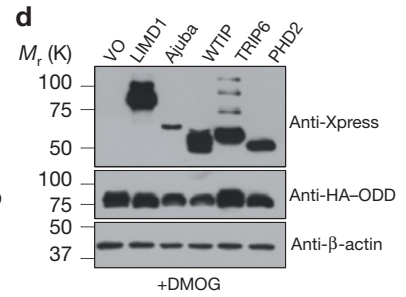

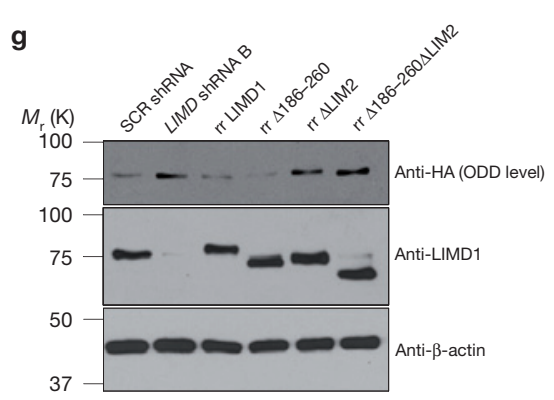
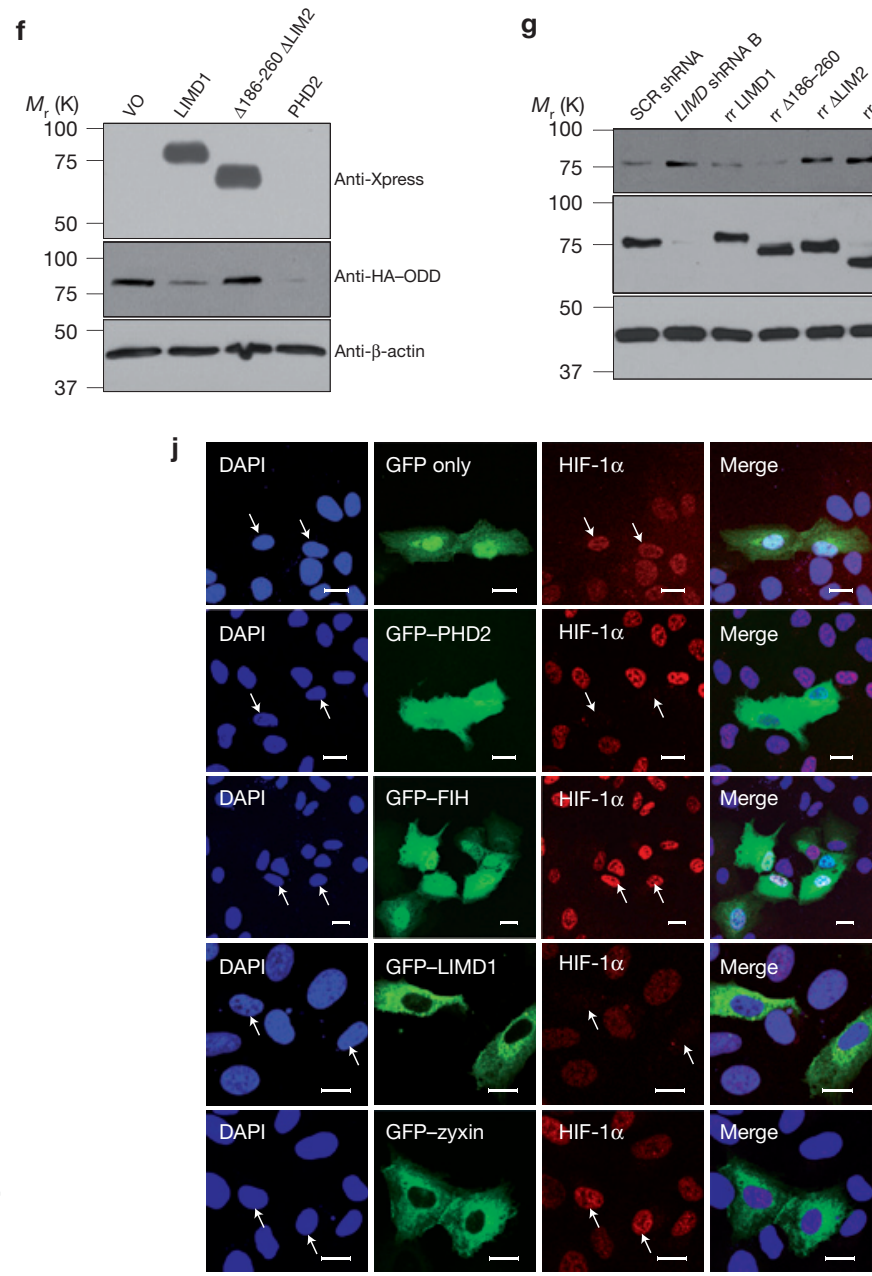

Figure 3 LIMD1-induced ODD degradation is dependent on PHD, proteasomal activities and PHD2/VHL binding. (a) Plasmids encoding Xpress-tagged LIMD1 or TRIP6 and haemagglutinin (HA)-HIF- $1 \alpha \mathrm{N}$ (amino acids 30-389), ODD (amino acids 390-652) or C-terminal (amino acids 630-826) domains were co-transfected into U2OS cells. Ectopic expression of LIMD1 (but not TRIP6) induces a specific reduction in ODD, but not in $\mathrm{N}$ - or C-terminal domains. (b) Plasmids encoding HIF-1 $\alpha \mathrm{N}-$, ODD or C-terminal domains were transfected into lentiviral shRNA B-mediated LIMD1-depleted and rescued U2OS cell lines. LIMD1 depletion resulted in stabilization of the ODD domain, denoted by the arrow. LIMD1 protein depletion had no effect on the $\mathrm{N}$ - or C-terminal HIF-1 $\alpha$ domains. SCR, scrambled. (c) Plasmids encoding the indicated family members and PHD2 as a positive control were co-transfected into U2OS cells with HA-ODD. PHD2 expression resulted in degradation of the ODD, whereas TRIP6 expression (negative control) did not. (d,e) The ability to induce ODD degradation is dependent on hydroxylase and $26 \mathrm{~S}$ proteasome activity, as ODD degradation was not observed when cells were treated with the PHD2 inhibitor DMOG (d) or the 26S proteasome inhibitor MG132 (e). (f) Plasmids encoding Xpress-tagged wild-type LIMD1 or LIMD1 unable to bind PHD2 and VHL ( $\Delta 186-260 \Delta$ LIM2) were co-transfected

with HA-ODD into U2OS cells. (g) U2OS cell lines stably expressing RNAi-resistant LIMD1 or LIMD1 internal mutants that are unable to bind PHD2 ( $\Delta 186-260)$, VHL $(\Delta \mathrm{LIM} 2)$ or both $(\Delta 186-260 \Delta \mathrm{LIM} 2)$ in a lentiviral-driven LIMD1 shRNA B-depleted background were transfected with plasmids encoding HA-ODD. (h) The same stably expressing lentiviral shRNA B cell lines were transfected with synthetic pGL3-HRE-luc and TK-Renilla (for normalization). LIMD1 depletion caused an increase in HRE transcriptional activation, which was repressed with re-expression of an rrLIMD1. Rescue with rrLIMD1 unable to bind PHD2 and VHL $(\Delta 186-260 \Delta L I M 2)$ increased in HRE transcriptional activation to the same extent as LIMD1 depletion. Data are mean \pm s.e.m., $n=3$ independent experiments, ${ }^{*} P<0.005,{ }^{*} P<0.05$. (i) Reporter analysis as in $\mathbf{h}$ was also carried out in hypoxia. (j) U2OS cells were transiently transfected with GFP vector only, GFP-PHD2, GFP-FIH, GFP-LIMD1 and GFP-zyxin. At $44 \mathrm{~h}$ following transfection, cells were exposed to $1 \% \mathrm{O}_{2}$ for $4 \mathrm{~h}$. Cells were then fixed and immunostained for endogenous HIF- $1 \alpha$ and nuclear stained using 4,6-diamidino-2-phenylindole (DAPI). Expression of LIMD1 reduced HIF- $1 \alpha$ expression levels and inhibited its nuclear accumulation; this was comparable in effect to GFP-PHD2. GFP-VO and GFP-FIH do not affect HIF- $1 \alpha$ stability or localization. Scale bars, $20 \mu \mathrm{m}$. 


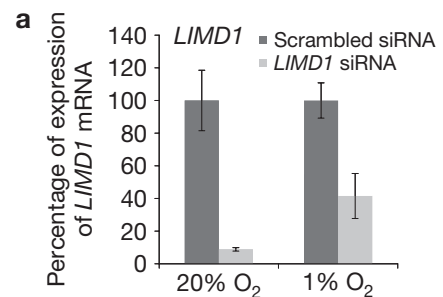

C

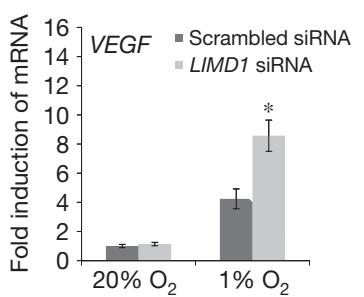

b

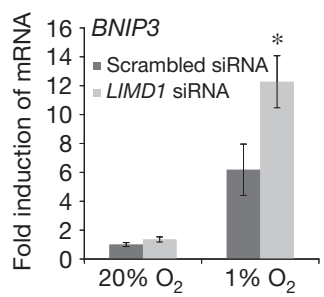

d

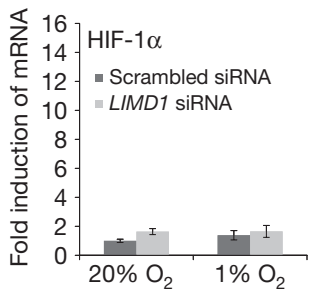

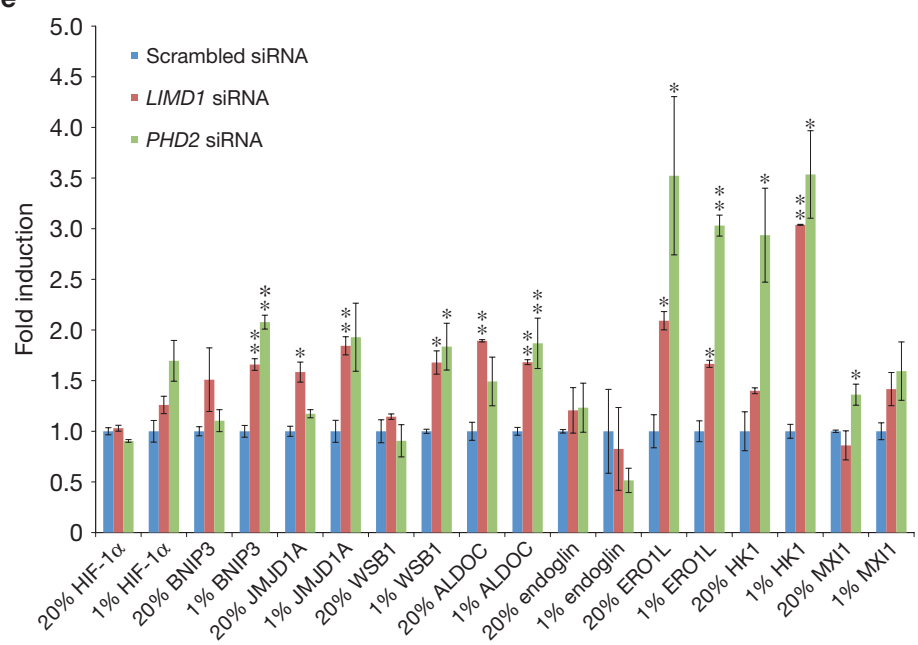

Figure 4 Depletion of endogenous LIMD1 induces expression of endogenous HIF-1-targeted genes. (a) LIMD1 mRNA levels were quantified to confirm RNAi-mediated depletion. LIMD1 mRNA levels were significantly reduced (in both normoxia and hypoxia) following LIMDI siRNA transfection relative to cells transfected with the scrambled siRNA control. (b) LIMD1 depletion caused a significant elevation in hypoxic $B N I P 3$ mRNA levels in comparison with the scrambled control; however, no change in BNIP3 mRNA levels was observed in normoxia. (c) LIMD1 depletion induced an increase in hypoxic VEGF mRNA levels. (d) HIF-1 $\alpha$ mRNA levels were unaffected by LIMD1 depletion, in normoxia and

extra HIF-1 $\alpha$ that is being hydroxylated and degraded through LIMD1-uncomplexed PHD2/VHL.

Using a previously described immunofluorescence assay to detect HIF-1 $\alpha$ (ref. 30), we examined the effect of ectopic LIMD1 on endogenous HIF-1 $\alpha$ protein levels in vivo. Expression of green fluorescent protein (GFP)-LIMD1 induced a reduction in HIF-1 $\alpha$ expression and inhibited nuclear accumulation (Fig. 3j), similar to expression of GFP-PHD2 (Fig. 3j). As further controls, expression of GFP only and factor inhibiting HIF (FIH) (ref. 31) had no effect on HIF- $1 \alpha$ expression level or localization (Fig. $3 j$ ). We believe that the overexpression of LIMD1 enhances formation of the complex, concentrating and recruiting any residually active PHD2 and VHL to HIF, thus facilitating its degradation. Family member zyxin, which does not bind VHL or PHD2, was used as a negative control (Fig. 3j, bottom panel), showing that the ability of a protein to engage PHD2 and VHL is critical for HIF-1 $\alpha$ degradation.

To further explore the physiological consequences of LIMD1 loss we examined the effect of depletion of endogenous LIMD1 on endogenous HIF-target genes (Fig. 4). siRNA-mediated LIMD1 depletion in U2OS cells resulted in a significant increase in both BNIP3 and VEGF messenger RNA levels in hypoxia (Fig. 4a-d). These responses were further validated in HEK293 cells for an extra set of HIF-responsive genes and with the inclusion of siRNA-targeted PHD2 depletion as a further control (Fig. 4e). Of note, there were clear gene-specific responses to LIMD1 and PHD2 depletion in both normoxia and hypoxia (Fig. 4e), and also differences in responses of genes between U2OS and HEK293 cell lines (Fig. 4a,b, compare BNIP3 in normoxia on LIMD1 depletion). One possible explanation for this could be the phosphorylation status (Supplementary Fig. S3b) and thus possible transcriptional activity of HIF-1 between these cell lines and also activity

hypoxia. (e) qRT-PCR analysis of endogenous HIF-1 $\alpha$ target genes in HEK293 cells depleted of LIMD1 and PHD1 by RNAi and subjected to $24 \mathrm{~h}$ at $20 \%$ or $1 \% \mathrm{O}_{2}$. Data shown as fold increase when compared with scrambled control normalized to unity. Data are mean \pm s.d., $n=3$ replicates, ${ }^{*} P<0.005,{ }^{* *} P<0.05$. BNIP3, BCL2/adenovirus E1B $M_{\mathrm{r}} 19 \mathrm{~K}$ interacting protein 3; VEGF, vascular endothelial growth factor $A$; JMJD1A, jumonji domain containing $1 A$; WSB1, WD repeat and SOCS box containing 1; ALDOC, aldolase C, fructose-bisphosphate; ERO1L, endoplasmic reticulum oxidoreductin-1-like; HK1, hexokinase 1; MXI1, MAX-interacting protein 1.

towards specific responsive genes discussed above. Furthermore, a similar observation was recently shown in ref. 32 , with the same unresponsiveness of endogenous HIF-1-responsive genes on increase in HIF-1 $\alpha$ level through depletion of MCM3 in normoxia, but increased responsiveness in hypoxia (see Fig. $2 \mathrm{k}$ in ref. 32). Taken together, these results indicate that reduction of endogenous LIMD1 is sufficient to increase HIF- $1 \alpha$ levels, HIF transcriptional activity and expression of endogenous HIF target genes.

In summary, we have shown that the Ajuba LIM proteins, particularly LIMD1, act to facilitate assembly of a PHD2-LIMD1-VHL protein complex that bridges the activities of PHD2 and VHL to enable efficient modification and degradation of HIF-1 $\alpha$. These findings suggest a new level in the regulation of HIF- $1 \alpha$ whereby hydroxylation and ubiquitylation are intimately associated enzymatic activities in a complex, allowing for efficient and rapid post-translational modification of HIF and its subsequent rapid degradation. Such a model allows for further regulation of HIF- $1 \alpha$ through regulation of complex association/formation (Fig. 5).

HIF modification is a critical regulatory step in the cellular response to low $\mathrm{O}_{2}$. Such a step requires tight, adaptive and diverse regulation to enable multiple signalling pathways to impinge on the hypoxic response with further levels of regulation. LIMD1, Ajuba and WTIP are unique among a growing group of PHD/HIF-1 $\alpha$ binding proteins (Siah1/2, FKBP38 and MCM family ${ }^{32-35}$ ) as they are the only regulators to simultaneously bind PHDs and VHL. Furthermore, given differential binding to the three PHDs, the operation of such complexes could potentially explain the fundamental paradox of how the simple biochemical/kinetic properties of the hydroxylase-ubiquitin ligase couple may be adapted to provide the flexibility necessary for physiological oxygen homeostasis. 
a

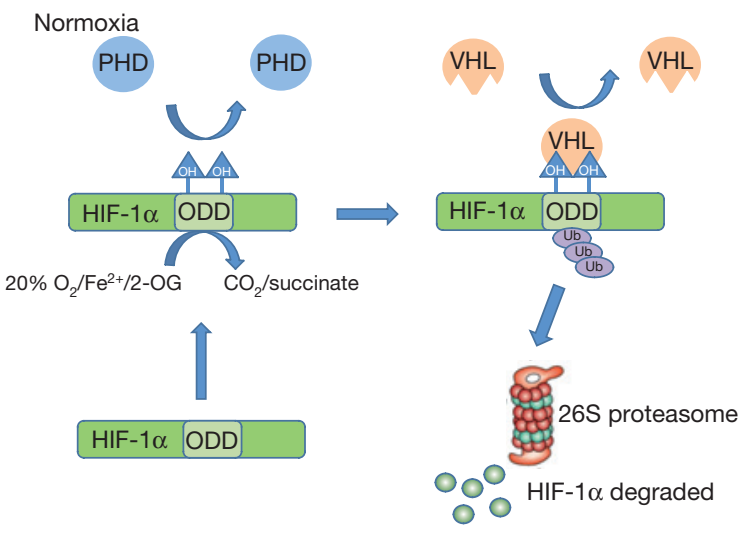

c

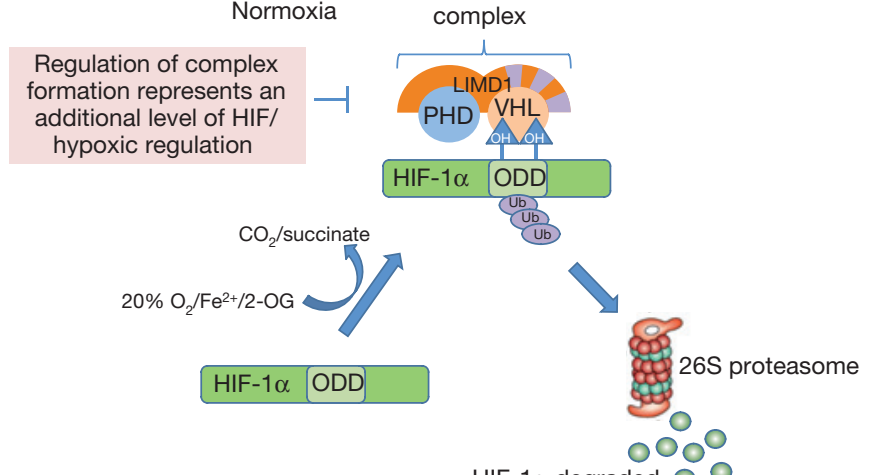

HIF-1 $\alpha$ degraded $\bigcirc \bigcirc$

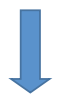

e

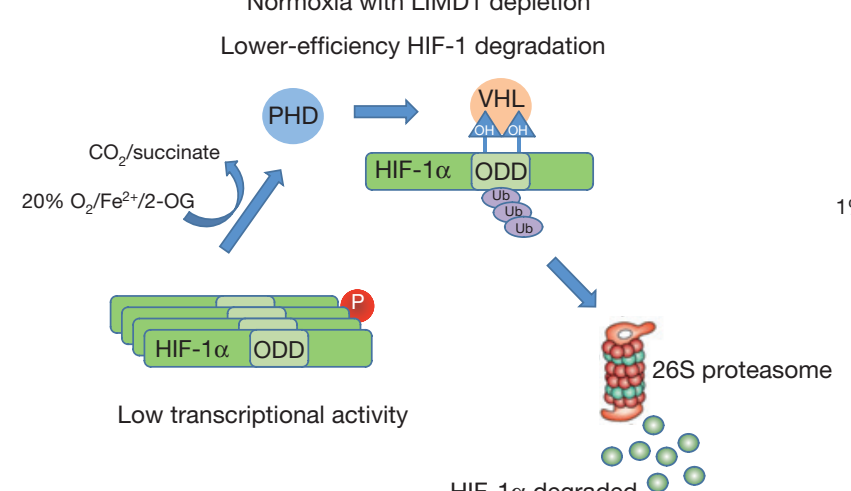

HIF-1 $\alpha$ degraded $\bigcirc$ b

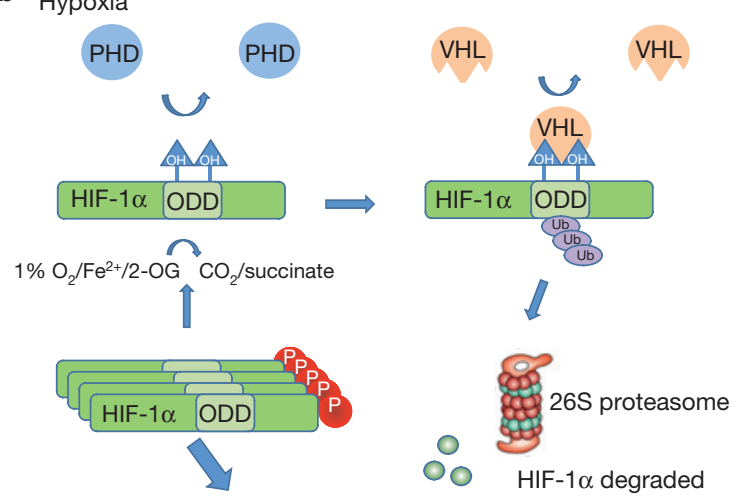

Transcription HRE genes

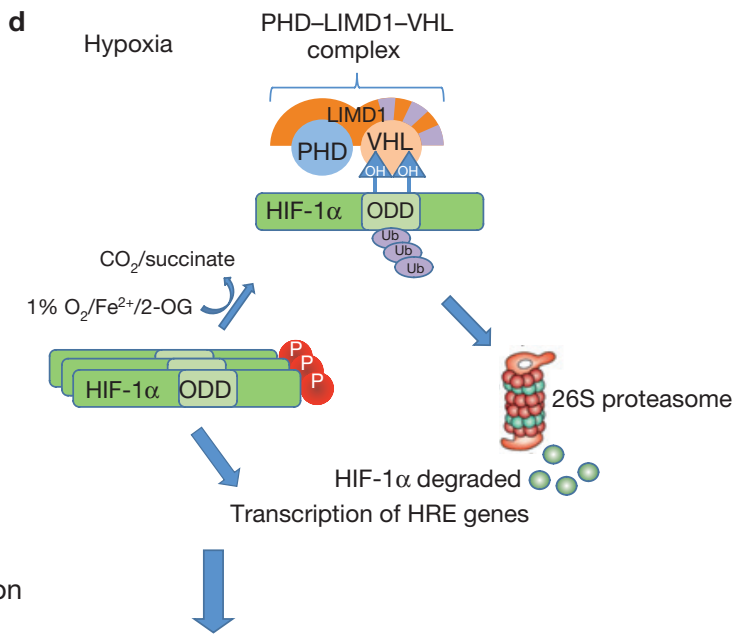

f

Hypoxia with LIMD1 depletion

Lower-efficiency HIF-1 degradation

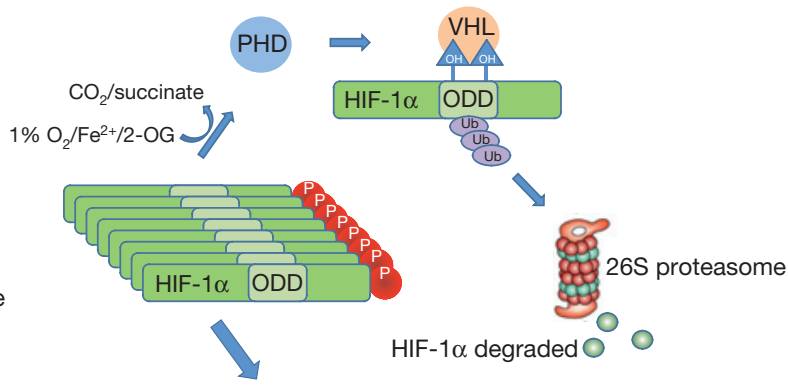

Transcription of HRE genes
Figure 5 Proposed model of LIMD1-mediated/dependent degradation of HIF-1 $\alpha .(\mathbf{a}, \mathbf{b})$ The current model depicts the prolyl hydroxylation and subsequent VHL-mediated polyubiquitylation (Ub) as distinct and separate processes. Under hypoxic $\left(1 \% \mathrm{O}_{2}\right)$ conditions the activity of PHD and thus subsequent hydroxylation/VHL-mediated ubiqutylation is significantly reduced, resulting in stabilization of HIF- $1 \alpha$, which then, dependent on cell type and conditions, becomes phosphorylated and transcriptionally active. (c) Our proposed new model suggests that LIMD1 interacts with PHD2 through its pre-LIM region and VHL through the LIM domains to link the proteins into one complex. Thus, LIMD1, Ajuba and WTIP may promote HIF-1 $\alpha$ degradation by the proteasome, by increasing the physical proximity of the enzymatic components responsible for hydroxylation and ubiquitylation. For simplicity, other proteins involved in regulating HIF $\alpha$ stability have not been depicted in this model, including elongin $\mathrm{B}, \mathrm{C}$ and cullin 2 within the $\mathrm{VHL}$-elongin B-elongin C complex and other Ajuba/zyxin family members. (d) Under hypoxia we propose that LIMD1 still engages an active pool of PHD2 together with VHL to enable a degree of HIF-1 $\alpha$ degradation in hypoxia. However, this is not sufficient to prevent build-up and the classic HIF-driven transcriptional response. Furthermore, this complex may be critical for adaptation to hypoxia. (e) In normoxia with LIMD1 depletion we propose that this results in a disengagement of PHD2 from VHL, resulting in reduced efficiency of HIF-1 $\alpha$ modification and degradation. Under such conditions there is a significant increase of HIF- $1 \alpha$ levels, of which a small proportion may become phosporylated and thus transcriptionally active. (f) In hypoxic conditions with LIMD1 depletion (compare $\mathbf{d}$ and $\mathbf{f}$ ) we see a greatly exaggerated increase in HIF-1 $\alpha$, which we believe indicates a loss of the LIMD1-dependent HIF- $1 \alpha$ degradation that occurs under hypoxic conditions. Our model does not rule out the existence of LIMD1-independent PHD/VHL-driven HIF- $1 \alpha$ degradation pathways, but proposes that these are significantly less efficient than the LIMD1-dependent one. 


\section{METHODS}

Methods and any associated references are available in the online version of the paper at http://www.nature.com/naturecellbiology

Note: Supplementary Information is available on the Nature Cell Biology website

\section{ACKNOWLEDGEMENTS}

We thank R. Layfield, T. Hagen, M. Cockman and S. Kristjansdottir for reagents and technical advice/assistance. K.S.B. is supported by a Biotechnology and Biological Sciences Research Council Doctorate Training Award. V.J. and D.E.F. were supported by funding from the Biotechnology and Biological Sciences Research Council (BB/F006470/1 and BB/I007571/1) awarded to T.V.S.

\section{AUTHOR CONTRIBUTIONS}

D.E.F., K.S.B., V.J., T.M.W. and T.V.S. designed experiments and wrote the paper. M.M., S.C.K.W., D.C-T. and T.E.P. carried out experiments. Y.F., P.J.R., S.I., J.B. and G.D.L. provided reagents. P.J.R. and G.D.L. contributed to experimental design and writing and editing the paper. T.V.S. initiated and managed this investigation.

\section{COMPETING FINANCIAL INTERESTS}

The authors declare no competing financial interests.

Published online at http://www.nature.com/naturecellbiology

Reprints and permissions information is available online at http://www.nature.com/ reprints

1. Epstein, A. C. et al. C. elegans EGL-9 and mammalian homologs define a family of dioxygenases that regulate HIF by prolyl hydroxylation. Cell 107, 43-54 (2001).

2. Bruick, R. K. \& McKnight, S. L. A conserved family of prolyl-4-hydroxylases that modify HIF. Science 294, 1337-1340 (2001).

3. Jaakkola, P. et al. Targeting of HIF- $\alpha$ to the von Hippel-Lindau ubiquitylation complex by $\mathrm{O}_{2}$-regulated prolyl hydroxylation. Science 292 , 468-472 (2001).

4. Ivan, M. et al. HIF $\alpha$ targeted for VHL-mediated destruction by proline hydroxylation: implications for $\mathrm{O}_{2}$ sensing. Science 292, 464-468 (2001).

5. Yu, F., White, S. B., Zhao, Q. \& Lee, F. S. HIF-1 $\alpha$ binding to VHL is regulated by stimulus-sensitive proline hydroxylation. Proc. Natl Acad. Sci. USA 98, 9630-9635 (2001).

6. Masson, N., Willam, C., Maxwell, P. H., Pugh, C. W. \& Ratcliffe, P. J. Independent function of two destruction domains in hypoxia-inducible factor- $\alpha$ chains activated by prolyl hydroxylation. EMBO J. 20, 5197-5206 (2001).

7. Sharp, T. V. et al. LIM domains-containing protein 1 (LIMD1), a tumour suppressor encoded at chromosome $3 \mathrm{p} 21.3$, binds pRB and represses E2F-driven transcription. Proc. Natl Acad. Sci. USA 101, 16531-16536 (2004).

8. Sharp, T. V. et al. The chromosome 3p21.3-encoded gene, LIMD1, is a critical tumour suppressor involved in human lung cancer development. Proc. Natl Acad. Sci. USA 105, 19932-19937 (2008).

9. Schmeichel, K. L. \& Beckerle, M. C. The LIM domain is a modular protein-binding interface. Cell 79, 211-219 (1994).

10. Kadrmas, J. L. \& Beckerle, M. C. The LIM domain: from the cytoskeleton to the nucleus. Nat. Rev. Mol. Cell Biol. 5, 920-931 (2004).

11. Appelhoff, R. J. et al. Differential function of the prolyl hydroxylases PHD1, PHD2, and PHD3 in the regulation of hypoxia-inducible factor. J. Biol. Chem. 279, 38458-38465 (2004).

12. Berra, E. et al. HIF prolyl-hydroxylase 2 is the key oxygen sensor setting low steady-state levels of HIF-1 $\alpha$ in normoxia. EMBO J. 22, 4082-4090 (2003).

13. Rankin, E. B. \& Giaccia, A. J. The role of hypoxia-inducible factors in tumorigenesis. Cell Death Differ. 15, 678-685 (2008).
14. Huggins, C. J. \& Andrulis, I. L. Cell cycle regulated phosphorylation of LIMD1 in cell lines and expression in human breast cancers. Cancer Lett. 267, 55-66 (2008).

15. Ivan, M. et al. Biochemical purification and pharmacological inhibition of a mammalian prolyl hydroxylase acting on hypoxia-inducible factor. Proc. Natl Acad. Sci. USA 99, 13459-13464 (2002).

16. Feng, Y. \& Longmore, G. D. The LIM protein Ajuba influences interleukin-1-induced $\mathrm{NF}-\mathrm{\kappa B}$ activation by affecting the assembly and activity of the protein kinase Czeta/p62/TRAF6 signalling complex. Mol. Cell Biol. 25, 4010-4022 (2005).

17. Iliopoulos, O., Ohh, M. \& Kaelin, W. G. Jr pVHL19 is a biologically active product of the von Hippel-Lindau gene arising from internal translation initiation. Proc. Nat Acad. Sci. USA 95, 11661-11666 (1998).

18. Schoenfeld, A., Davidowitz, E. J. \& Burk, R. D. A second major native von Hippel-Lindau gene product, initiated from an internal translation start site, functions as a tumour suppressor. Proc. Natl Acad. Sci. USA 95 8817-8822 (1998).

19. Liu, W., Xin, H., Eckert, D. T., Brown, J. A. \& Gnarra, J. R. Hypoxia and cell cycle regulation of the von Hippel-Lindau tumour suppressor. Oncogene 30 21-31 (2011).

20. Feng, Y. et al. A multifunctional lentiviral-based gene knockdown with concurrent rescue that controls for off-target effects of RNAi. Genom. Proteom. Bioinform. 8 238-245 (2010).

21. Chan, D. A., Sutphin, P. D., Yen, S. E. \& Giaccia, A. J. Coordinate regulation of the oxygen-dependent degradation domains of hypoxia-inducible factor $1 \alpha$. Mol. Cell Biol. 25, 6415-6426 (2005).

22. Stiehl, D. P. et al. Increased prolyl 4-hydroxylase domain proteins compensate for decreased oxygen levels. Evidence for an autoregulatory oxygen-sensing system. J. Biol. Chem. 281, 23482-23491 (2006).

23. Ginouves, A., Ilc, K., Macias, N., Pouyssegur, J. \& Berra, E. PHDs overactivation during chronic hypoxia 'desensitizes' HIF $\alpha$ and protects cells from necrosis. Proc. Natl Acad. Sci. USA 105, 4745-4750 (2008).

24. Tan, M. et al. SAG/ROC2/RBX2 is a HIF-1 target gene that promotes HIF-10 ubiquitination and degradation. Oncogene 27, 1404-1411 (2008).

25. Wang, G. L. \& Semenza, G. L. Characterization of hypoxia-inducible factor 1 and regulation of DNA binding activity by hypoxia. J. Biol. Chem. 268, 21513-21518 (1993).

26. Berra, E. et al. Signaling angiogenesis via p42/p44 MAP kinase and hypoxia Biochem. Pharmacol. 60, 1171-1178 (2000).

27. Minet, E., Michel, G., Mottet, D., Raes, M. \& Michiels, C. Transduction pathways involved in Hypoxia-Inducible Factor-1 phosphorylation and activation. Free Radic. Biol. Med. 31, 847-855 (2001).

28. Richard, D. E., Berra, E., Gothie, E., Roux, D. \& Pouyssegur, J. p42/p44 mitogen-activated protein kinases phosphorylate hypoxia-inducible factor $1 \alpha$ (HIF-1 $\alpha$ ) and enhance the transcriptional activity of HIF-1. J. Biol. Chem. 274, 32631-32637 (1999).

29. Huang, L. E., Gu, J., Schau, M. \& Bunn, H. F. Regulation of hypoxia-inducible factor $1 \alpha$ is mediated by an $\mathrm{O}_{2}$-dependent degradation domain via the ubiquitin-proteasome pathway. Proc. Natl Acad. Sci. USA 95, 7987-7992 (1998).

30. Metzen, E. et al. Intracellular localisation of human HIF-1 $\alpha$ hydroxylases: implications for oxygen sensing. J. Cell Sci. 116, 1319-1326 (2003).

31. Lando, D. et al. FIH-1 is an asparaginyl hydroxylase enzyme that regulates the tran scriptional activity of hypoxia-inducible factor. Genes Dev. 16, 1466-1471 (2002).

32. Hubbi, M. E., Luo, W., Baek, J. H. \& Semenza, G. L. MCM proteins are negative regulators of hypoxia-inducible factor 1 . Mol. Cell 42, 700-712 (2011).

33. Nakayama, K. \& Ronai, Z. Siah: new players in the cellular response to hypoxia. Cell Cycle 3, 1345-1347 (2004)

34. Barth, S. et al. The peptidyl prolyl cis/trans isomerase FKBP38 determines hypoxia-inducible transcription factor prolyl-4-hydroxylase PHD2 protein stability. Mol. Cell Biol. 27, 3758-3768 (2007).

35. Barth, S. et al. Hypoxia-inducible factor prolyl-4-hydroxylase PHD2 protein abundance depends on integral membrane anchoring of FKBP38. J. Biol. Chem 284, 23046-23058 (2009). 


\section{METHODS}

Cell culture and transfections. Human cell lines (U2OS, HeLa, HEK293, HEK293T) were cultured in DMEM (PAA Laboratories), supplemented with $10 \%(\mathrm{v} / \mathrm{v})$ fetal calf serum (Sigma), $50 \mathrm{U} \mathrm{ml}^{-1}$ penicillin and $50 \mathrm{~g} \mathrm{ml}^{-1}$ streptomycin (Sigma). Hypoxic treatment at $1 \% \mathrm{O}_{2}$ was achieved using a ProOx 110 (BioSpherix) controller and chamber. Proteasomal inhibition was with $10 \mu \mathrm{M}$ MG-132 (Enzo) for $4 \mathrm{~h}$. 2-OG dioxygenase inhibition was achieved using $1 \mathrm{mM}$ DMOG for $4 \mathrm{~h}$. For transfected co-immunoprecipitation assays, siRNA and qRT-PCR, $2 \times 10^{5} \mathrm{U} 2 \mathrm{OS}$ or $4 \times 10^{5}$ HEK293T cells were plated into six-well plates. For transient transfection and reporter assays, $5 \times 10^{4} \mathrm{U} 2 \mathrm{OS}$ or $8 \times 10^{4} \mathrm{U} 2 \mathrm{OS}$ shRNA lines were plated into 12-well plates. U2OS cells were transfected using Genejuice (Merck Biosciences) or TransIT LT1 (Mirus Bio) and HEK293T cells were transfected using TransIT 293. siRNA was transfected using DharmaFECT Duo or Dharma FECT 1 (Dharmacon) transfection reagents. Transfections were as per manufacturer's instructions.

siRNA and shRNA stable line production. The siRNA sequence (Sigma Mission siRNA) against LIMD1 was as follows: 5'-GCAAGGAGGUCUUCCAAGA-3' (assay code SASI_Hs01_00095038).

The shRNA sequences against LIMD1 were as follows. shRNA A: $h$ LIMD1 shRNA 3' UTR, 5'-GCAGAATGGCTGCAAATTTAA-3'. shRNA B: hLIMD1 shRNA 5' UTR, 5'-GTCTGCAGCATGGATAAGTA-3'.

U2OS stable cell lines were generated using the lentiviral shRNA-driven LIMD1 knockdown and rescue system, as previously described ${ }^{36}$. The gene delivery and production system was as previously described ${ }^{37}$.

Plasmids. Generation of pcDNA4/HisMax (Invitrogen) Xpress-LIMD1 and LIMD1 deletion mutants and pcDNA3.1 PHD1, 2, 3 have previously been described $^{38,39}$. pcDNA3.1 (Invitrogen) HIF-1 $\alpha$, FIH, VHL, HA-enhanced GFP (eGFP)-ODD, N-terminal HIF-1 $\alpha$, C-terminal HIF- $1 \alpha$ and pGL3-HRE plasmids were kindly donated by Thilo Hagen, National University of Singapore. pcDNA3.1 mLIMD1 and mLIMD1 deletion mutants, and pFLRu Scr-GFP, hLIMD1 shRNA-GFP, hLIMD1 shRNA rrhLIMD1-FH and hLIMD1 shRNA rrhLIMD1 $\triangle$ LIM2-FH were donated by Y. Feng and G. Longmore, Washington University ${ }^{20,36}$. pGEX4T-1-LIMD1 and pEGFP-LIMD1 were generated as follows. LIMD1 complementary DNA was excised from pcDNA4/HisMax-LIMD1 using EcoRI and SalI restriction endonucleases and ligated into similarly cut pGEX4T-1 (Amersham) and pEGFP $\mathrm{Cl}+1$ vector (donated by S. Dawson, University of Nottingham) respectively. Xpress-tagged PHD1, 2 and 3 were generated by PCR amplification of cDNA from pcDNA3.1-PHD1,2 and 3, incorporating flanking BamHI and EcoRI sites (for PHD3 BglII and EcoRI) and cloned into the pcDNA4/HisMax vector (Invitrogen). pEGFP-PHD1, 2 and 3 were generated by restriction digest from pcDNA4/HisMax into the pEGFP vector. Xpress-tagged LIMD1 conserved-region deletion mutants $\Delta 112-123, \Delta 140-166, \Delta 186-260$, $\Delta 239-260, \Delta 298-315, \Delta 342-369, \Delta 429-464, \Delta$ LIM2 (residues 537-591) and double mutant $\Delta$ 186-260- $\Delta$ LIM2 were generated from pcDNA4/HisMax LIMD1 through the QuikChange site-directed mutagenesis method (Stratagene). Using the same method, pFLRu- $h$ LIMD1 shRNA + rrhLIMD1 $\Delta 186-260-\mathrm{FH}$ and $\Delta$ LIM2-FHwere generated from template pFLRu- $h$ LIMD1 shRNA + rrLIMD1 and subsequently pFLRu- $h L I M D 1$ shRNA + rrhLIMD1 $\Delta 186-260 \Delta$ LIM2-FH was generated from $p F L R u-h L I M D 1$ shRNA + rrhLIMD1 $\Delta 186-260-\mathrm{FH}$.

Forward primer sequences for the mutagenesis of Xpress-tagged LIMD1 conserved-region deletion mutants were as follows: $\Delta 112-123,5^{\prime}$-CTTGCTGCCT CGACACAGCCCCCGTAC-3'; $\triangle 140-166,5^{\prime}$-CCATACCTGCATCCCTGTGAGGATC-3'; $\Delta 186-260, \quad 5^{\prime}$-GGAGACTATTATGACAACCTCTCCTTGGGCCTTTGGTCCACTGCCTCCTC-3'; $\Delta 239-260,5^{\prime}$-CTGAGCTCCAGCAGGGGCCTTTGGTCC-3'; $\Delta 298-315, \quad 5^{\prime}$-AGGACCCCTTCTGTGTCGGGGCTGGGGGGTG-3'; $\Delta 342-369,5^{\prime}$-CAGGATGGGCCCCCGAAGCCTGGCTGC-3'; $\Delta 429-464,5^{\prime}$-TCCCCTAGGGTAAGGAAGGCTGATTAC-3'; $\Delta$ LIM2 (residues 537-591), 5' -CTGGTTTCCAGCAGTCGGCTGACAGGGTGCTGGCCCCCAAGTGTGCAGCC-3'. Reverse primers were exact reverse complements of the forward primers.

Yeast two-hybrid screen. LIMD1 $\triangle 364-676 \mathrm{cDNA}$ was released from pcDNA4 /HisMax LIMD1 $\Delta 364-676$ vector and ligated into the similarly cut pAS2.1 bait vector. The resulting plasmid (pAS2.1-LIMD1- $\Delta 364-676)$ was pretransformed into the Saccharomyces cerevisiae PJ69-4a (MATa trp1-90 leu2-3,112 ura3-52 his3200 gal4 $\triangle$ gal80 $\triangle$ LYS2::GAL1-HIS3 GAL2-ADE2 met2::GAL7-lacZ) (14) reporter strain by using a modified lithium acetate protocol (see Yeast Protocols Handbook, BD Biosciences Clontech). Subsequently, this strain was cotransformed with the BC3 cDNA library (produced by using the Stratagene HybriZAP cDNA library kit) in the GAL4 DNA activation domain (GAL4AD) fusion 'prey' vector. Selection for positive colonies and cDNA clone isolation were as previously described ${ }^{40}$.

Sucrose-gradient fractionation. HEK293 were lysed in $25 \mathrm{mM}$ Tris- $\mathrm{HCl}$ at $\mathrm{pH}$ 7.4, $150 \mathrm{mM} \mathrm{KCl}, 0.5 \%$ NP-40, $2 \mathrm{mM}$ EDTA, $1 \mathrm{mM} \mathrm{NaF}, 0.5 \mathrm{mM}$ dithiothreitol supplemented with 'Complete' protease inhibitors (Roche) and PhosSTOP phosphatase inhibitors (Roche $)^{41}$. The cell lysate was rotated at $4{ }^{\circ} \mathrm{C}$ for $30 \mathrm{~min}$, centrifuged at 13,000 r.p.m. for $10 \mathrm{~min}$, and the cleared lysate applied to a $10-35 \%$ discontinuous sucrose gradient in polyallomer tubes. The gradient was centrifuged at 30,000 r.p.m. for $16.5 \mathrm{~h}$ (SW41Ti rotor, Optima LE- $80 \mathrm{~K}$ ultracentrifuge), and $500 \mu \mathrm{l}$ fractions were collected from the top of the gradient (fraction number 1) downwards to the bottom of the gradient (fraction number 17).

In vitro binding assays. pGEX4T-PHD2 and pGEX6P-LIMD1 were transformed into BL21 (DE3)-pLysS chemically competent bacteria. Cultures were grown for $3 \mathrm{~h}$ before induction with IPTG followed by a further $4 \mathrm{~h}$ growth at $37^{\circ} \mathrm{C}$. Bacteria were pelleted, freeze-thawed and lysed by sonication. Recombinant proteins were purified onto glutathione Sepharose 4B (GE Healthcare 17-0756-01). Recombinant LIMD1 was cleaved from the Sepharose using PreScission protease (GE Healthcare 270843 ) in binding buffer (50 mM Tris- $\mathrm{HCl}$ at $\mathrm{pH} 7.0,150 \mathrm{mM} \mathrm{NaCl}, 1 \mathrm{mM}$ EDTA, $1 \mathrm{mM}$ dithiothreitol). Equal amounts of the cleaved recombinant LIMD1 were incubated with either purified GST vector only or GST-PHD2 for $6 \mathrm{~h}$ at $4{ }^{\circ} \mathrm{C}$ and washed three times with binding buffer.

Immunoprecipitations, immunoblots and immunofluorescence. For coimmunoprecipitation of ectopically expressed proteins, cells were lysed by the addition of ice-cold RIPA buffer ( $150 \mathrm{mM} \mathrm{NaCl}, 1 \%$ (v/v) IGEPAL-630, 0.5\% (w/v) sodium deoxycholate, $0.1 \%$ (w/v) SDS, $50 \mathrm{mM}$ Tris, $\mathrm{pH}$ 8) supplemented with 'Complete' protease inhibitors (Roche) and PhosSTOP phosphatase inhibitors (Roche) and scraped using a cell scraper. Lysates were rotated at $4{ }^{\circ} \mathrm{C}$ for $20 \mathrm{~min}$ and centrifuged at 13,000 r.p.m. for $10 \mathrm{~min}$. The cleared lysate was rotated at $4{ }^{\circ} \mathrm{C}$ for $4 \mathrm{~h}$ with immunoprecipitation matrix (Santa Cruz sc-45042) previously conjugated to $2 \mu \mathrm{g}$ of immunoprecipitating antibody (anti-Xpress (Invitrogen), anti-V5 (AbD-Serotec), LIMD1 antibody (3F2/C6; ref. 39), anti-GFP (Roche)). The immunoprecipitation matrix-antibody complex was then washed three times with ice-cold RIPA, and protein complexes were eluted in $5 \times$ SDS-PAGE sample buffer.

For endogenous co-immunoprecipitations, cells were lysed in $1 \mathrm{ml}$ of RIPA buffer supplemented with 'Complete' protease and phosphatase inhibitors, rotated at $4{ }^{\circ} \mathrm{C}$ for $30 \mathrm{~min}$ and centrifuged at 13,000 r.p.m. for $10 \mathrm{~min}$. Cleared lysates were incubated for $5 \mathrm{~h}$ at $4^{\circ} \mathrm{C}$ with $5 \mu \mathrm{g}$ of mouse monoclonal VHL (BD Transduction Laboratories), PHD2 (Millipore) or matching $\mathrm{IgG}_{1 \mathrm{~K}}$ isotype control GFP (Roche) antibody that had been previously conjugated to immunoprecipitation matrix (Santa Cruz) overnight. The immunoprecipitation matrix-antibody complex was then washed three times with ice-cold RIPA/PBS $(50 \% \mathrm{v} / \mathrm{v})$ and protein complexes eluted in $5 \times$ SDS-PAGE sample buffer. SDS-PAGE and immunoblots were carried out using standard protocols.

For immunofluorescence, cells were fixed with $4 \%(\mathrm{v} / \mathrm{v})$ paraformaldehyde in PBS at room temperature for $5 \mathrm{~min}$ and permeabilized with $0.05 \%(\mathrm{v} / \mathrm{v})$ Triton $\mathrm{X}-100$ in PBS at room temperature with gentle agitation. Cells were washed with PBS, incubated in blocking buffer ( $3 \%$ (w/v) BSA, $1 \mathrm{mM} \mathrm{MgCl}, 1 \mathrm{mM} \mathrm{CaCl}{ }_{2}$ in $1 \times$ PBS) for $1 \mathrm{~h}$ at room temperature with gentle shaking and then washed in washing solution $\left(0.3 \%(\mathrm{w} / \mathrm{v}) \mathrm{BSA}, 1 \mathrm{mM} \mathrm{MgCl} 2,1 \mathrm{mM} \mathrm{CaCl}_{2}\right.$ in $\left.1 \times \mathrm{PBS}\right)$. Cells were incubated with anti - HIF-1 $\alpha$ (BD Transduction Laboratories, 1:500 dilution) primary antibody and an Alexafluor (Invitrogen, 1:1,000 dilution) secondary antibody.

Ubiquitylation assay. This assay was essentially carried out as described in ref. 32. Briefly: cells were transfected with expression vectors encoding V5-HIF-1 $\alpha$, Flag-ubiquitin, EV or Xpress-LIMD1. Cells were lysed, mixed and incubated for $1 \mathrm{~h}$ at $37^{\circ} \mathrm{C}$. Lysates were then immunoprecipitated with V5 antibody (AbD Serotec) followed by immunoblot with antibodies to HIF-1 $\alpha$ (BD Transduction Laboratories, 1:500 dilution) or Flag (Sigma Aldrich, 1:1,000).

Antibodies. A list of all primary and secondary antibodies and dilutions used can be found in Supplementary Tables S1 and S2 respectively.

Reporter assays. Cells were co-transfected in triplicate with pGL3-(6x)HRE-firefly luciferase and TK-Renilla luciferase reporters. $24 \mathrm{~h}$ post-transfection cells were lysed in $1 \times$ passive lysis buffer (Promega) and freeze-thawed. Luciferase activity was assayed using the dual luciferase reporter assay system (Promega) and TopCount scintillation and luminescence counter (Perkin Elmer).

Phosphatase assay. Cells were exposed to $1 \% \mathrm{O}_{2}$ for $16 \mathrm{~h}$ before lysis by scraping in RIPA buffer supplemented with 'Complete' protease and phosphatase inhibitors and $10 \mu \mathrm{M}$ MG-132. Cleared lysates were added to $2.5 \mu \mathrm{gHIF}-1 \alpha$ or control antibody conjugated to immunoprecipitation matrix. Immunoprecipitation of HIF-1 $\alpha$ was carried out for $4 \mathrm{~h}$ at $4{ }^{\circ} \mathrm{C}$ with rotation, followed by extensive washing with unsupplemented RIPA buffer. Dephosphorylation was carried out in $50 \mu$ lreaction volumes using 400 units of $\lambda$ protein phosphatase (NEB no P0753S) at $30^{\circ} \mathrm{C}$ for $60 \mathrm{~min}$ along with a control reaction that omitted the enzyme. The reaction was stopped by addition of $5 \times$ SDS-PAGE sample buffer. 
Quantitative RT-PCR. Transfected cells were incubated for $24 \mathrm{~h}$ at $1 \% \mathrm{O}_{2}$ or maintained at standard incubation conditions for normoxic controls. Duplicate wells were lysed for protein using $100 \mu \mathrm{l}$ passive lysis buffer (Promega) and RNA extracted using the RNAqueous micro kit (Ambion) for U2OS cells or High Pure RNA Isolation Kit (Roche) for HEK293T cells as per manufacturer's instructions RNA $(1 \mu \mathrm{g})$ pre-treated with DNase I (Invitrogen) was then reverse transcribed using oligo-dT primers and High Fidelity Reverse Transcriptase (Roche). To quantify the mRNA levels of target genes quantitative real-time PCR was conducted using $1 \times$ Power SYBR Green Mastermix (Applied Biosystems), $0.4 \mathrm{mM}$ forward and reverse primers, $2.5 \mu \mathrm{l}$ cDNA and $\mathrm{dH}_{2} \mathrm{O}$ to a final volume of $25 \mu \mathrm{l}$; reactions were run on an ABI7000 instrument (Applied Biosystems). U2OS cells were transfected with $80 \mathrm{nM}$ of scrambled control and LIMD1 siRNA. $40 \mathrm{~h}$ post-transfection, cells were incubated in normoxia $\left(20 \% \mathrm{O}_{2}\right)$ or hypoxia $\left(1 \% \mathrm{O}_{2}\right)$ for $8 \mathrm{~h}$. Cells were lysed and RNA extracted using the RNAqueous microRNA extraction kit (Ambion). Quantitative RT-PCR ( QRT-PCR) was carried out using gene-specific primers spanning an exon boundary and SYBR Green I. Data were normalized to the housekeeping genes RNA polymerase II and $\beta$-tubulin and analysed using the relative quantification method 2-[delta][detta]Ct. The list of qRT-PCR primer sequences used can be found as Supplementary Table S3.

Statistical analysis. For reporter assays, firefly luciferase values were normalized against Renilla luciferase values. Error bars are the s.e.m. of the normalized values.
Data shown are the mean \pm s.e.m. of three independent determinations. Significant difference was determined using the Student's $t$-test.

For qRT-PCR, data were normalized to the housekeeping genes $\beta$-tubulin and RNA polymerase II and relative quantification was determined by the comparative cross-over threshold method (2-[delta][delta]Ct $)$.

36. Langer, E. M. et al. Ajuba LIM proteins are snail/slug corepressors required for neural crest development in Xenopus. Dev. Cell 14, 424-436 (2008).

37. Naldini, L. et al. In vivo gene delivery and stable transduction of nondividing cells by a lentiviral vector. Science 272, 263-267 (1996).

38. Epstein, A. C. et al. C. elegans EGL-9 and mammalian homologs define a family of dioxygenases that regulate HIF by prolyl hydroxylation. Cell 107, 43-54 (2001).

39. Sharp, T. V. et al. LIM domains-containing protein 1 (LIMD1), a tumour suppressor encoded at chromosome 3p21.3, binds pRB and represses E2F-driven transcription. Proc. Natl Acad. Sci. USA 101, 16531-16536 (2004).

40. Sharp, T. V. et al. K15 protein of Kaposi's sarcoma-associated herpesvirus is latently expressed and binds to HAX-1, a protein with antiapoptotic function. J. Virol. 76, 802-816 (2002).

41. Beitzinger, M., Peters, L., Zhu, J. Y., Kremmer, E. \& Meister, G. Identification of human microRNA targets from isolated argonaute protein complexes. RNA Biol. 4, 76-84 (2007). 
a

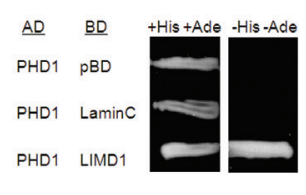

e
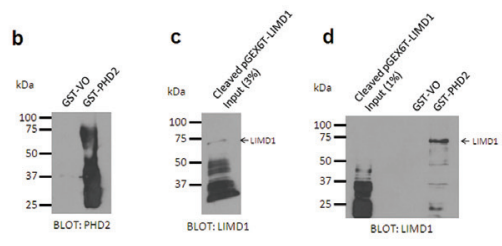

$\begin{array}{llllllllllllllll}1 & 2 & 3 & 4 & 5 & 6 & 7 & 8 & 9 & 10 & 11 & 12 & 13 & 14 & 15 & 16\end{array}$

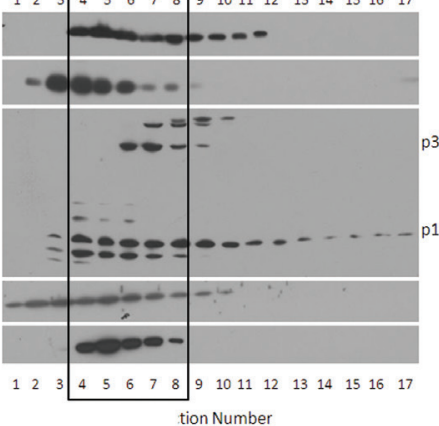

LIMD1

PHD2

p30

VHL

p19

Elongin B

Cullin 2

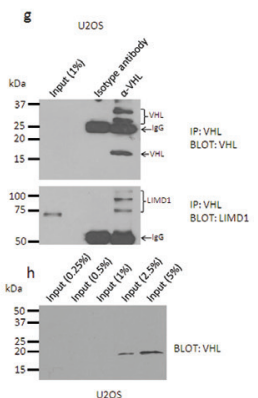

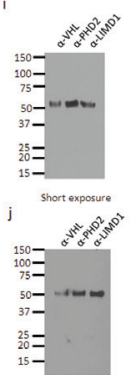

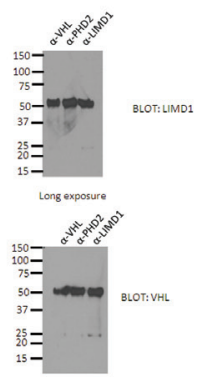

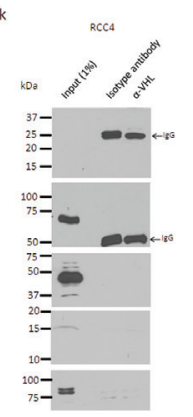
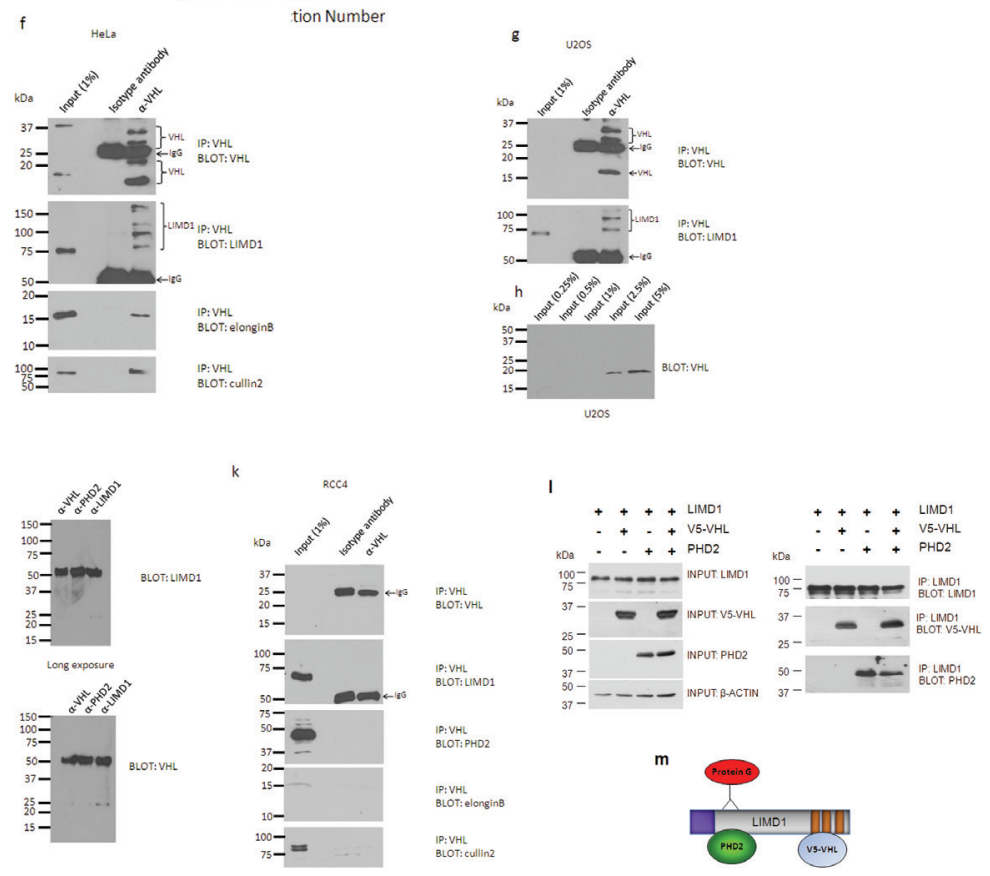

Figure S1 Identification of PHD binding to LIMD1 (a) A yeast 2 hybrid screen of a HeLa cDNA library with a LIMD1 $\triangle 364-676$ GAL4 DNAbinding domain identified a CDNA encoding full length PHD1 as binding protein. . AD, the indicated Gal4 activation domain fusion protein. BD, the indicated Gal4 DNA-binding domain fusion protein. BD-LaminC fusion used as negative control. Interation between the indicated fusion proteins was assayed via auxotrphy for amino acids His and Ade with growth on media lacking these two (-His, -Ade) indicative of specific interaction. (b) recombinant GST vector only or GST-PHD2 immobilised on glutathione beads were incubated with (c) PreScission Protease cleaved and purified recombinant LIMD1 $(\sim 73 \mathrm{kDa})$ in an in vitro direct binding assay (d), which demonstrated that LIMD1 and PHD2 directly interact. (e) Sucrose gradient analysis of LIMD1, PHD2, VHL, elonginB and Cullin 2. HEK 293 cell lysates were passed through a sucrose gradient and multiple fractions recovered. Fractions were taken from the top (\#1) to the bottom of the gradient (\#17). Protein content of each fraction was assayed by immunoblotting with the indicated antibodies. LIMD1 fractionated in the same common fraction(s) as PHD2, VHL (both p19 and p30 isoforms), elongin B and cullin 2 (black boxed area). Endogenous VHL and LIMD1 co-immunoprecipitate. VHL was endogenously immunoprecipitated from (f) HeLa and (g) U2OS cell lysates

and endogenous LIMD1 co-immunoprecipitated from both lysates. In the HeLa lysates endogenous elonginB and cullin2 also co-immunoprecipitated. (h) Low level expression of VHL in U2OS resulted in no detected with $1 \%$ input loaded. However, with $2.5 \%$ input or greater detection was achieved. (i) As controls for the possibility of non-specific cross reactivity of antibodies the antibody conjugated matrices alone were probed with anti-LIMD1 and (j) anti-VHL antibodies. Only the heavy and light chains were detected with the secondary antibody, demonstrating the interactions observed in the immunoprecipitations (Fig. 1b, c) were specific. (k) Similar immunopreciptations (as in Fig. 1c) were repeated in the VHL negative RCC4 cell lines as a negative control. No cross reactive/co-precipitated bands could be detected therefore further demonstrating the specificity of VHL associated LIMD1 and PHD2 in HEK 293 cells. LIMD1 non-competitively interacts with PHD2 and VHL and enhances their association. (I) LIMD1 expression plasmid was co-transfected into U2OS cells with V5-VHL and pcDNA3 PHD2 individually and in combination. LIMD1 was immunoprecipitated using an Xpress mAb. PHD2 and VHL co-immunprecipitated with LIMD1 individually and when co-expressed, with neither impairing the ability of LIMD1 to interact with the other. (m) Schematic representation illustrating the ability of LIMD1 to simultaneously interact with PHD2 and VHL within a protein complex. 
a

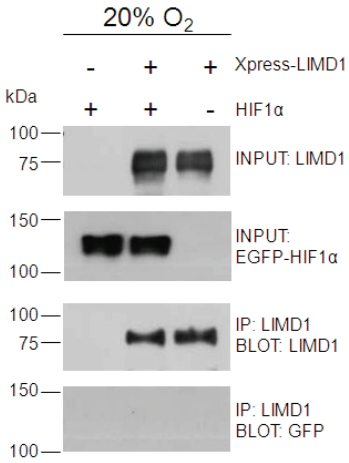

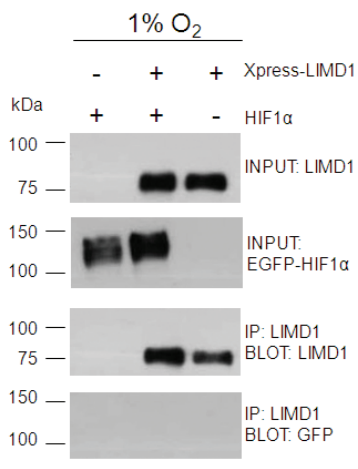

b

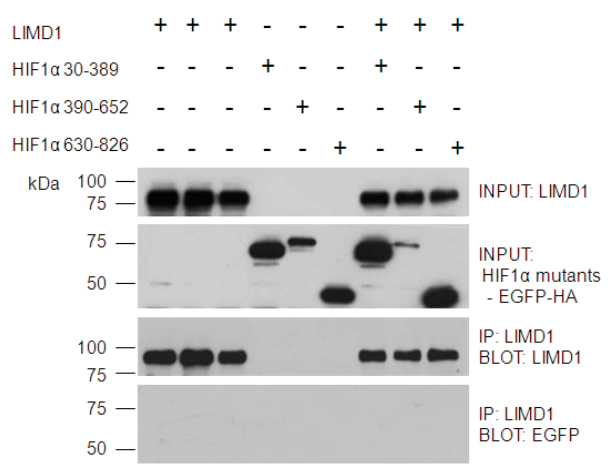

C

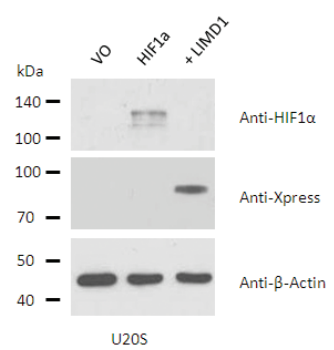

Figure S2 HIF1 $\alpha$ does not co-IP with LIMD1 in vivo. (a) Xpress-LIMD1 was co-transfected into U2OS cells with EGFP-HIF1 $\alpha$. LIMD1 was immunoprecipitated using an Xpress mAb, following hypoxic $\left(1 \% \mathrm{O}_{2}\right)$ or normoxic $\left(20 \% \mathrm{O}_{2}\right)$ incubation for 16 hours. HIFl $\alpha$ does not interact with LIMD1. (b) Xpress-LIMD1 was further co-transfected with the $\mathrm{N}$ - (aa30-389), ODD (390-652), or C- terminal (630-826) domains of HIF $1 \alpha$ all tagged with EGFP. Cells were incubated in hypoxia for 16 hours to induce ODD stability and LIMD1 was immunoprecipitated with an Xpress mAb. None of the transfected EGFP-HIF $1 \alpha$ domains were found to interact with LIMD1. (c) LIMD1 expression decreases HIF1 $\alpha$ protein levels. Reporter cell extracts expressing Xpress tagged vector only and LIMD1 were co-transfected with HIF1 $\alpha$ into U2OS cells. The indicted reporter cell lysates from Fig. $2 \mathrm{~b}$ western blotted with the indicated antibodies. 


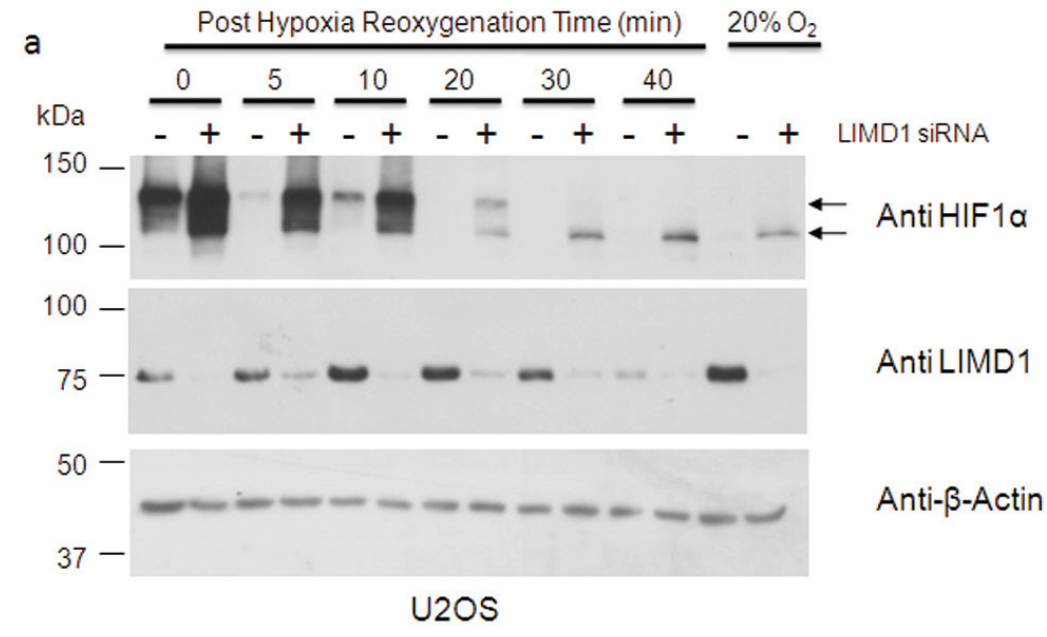

b

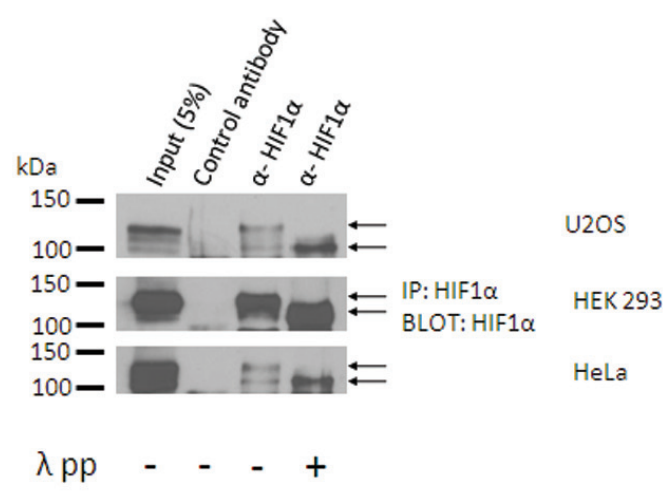

Figure S3 LIMD1 loss retards HIF1 $\alpha$ degradation following reoxygenation. (a) Depletion of LIMD1 in U2OS cells with 80nM siRNA caused a stabalisation of HIF1 $\alpha$ protein levels following 32 hours post transfection, cells were incubated in normoxia or hypoxia for 16 hours. Cells were taken out of hypoxia and into $20 \% \mathrm{O}_{2}$ for the indicated times prior to lysis. LIMD1 loss retards reoxygenation mediated degradation of HIFl $\alpha$. Furthermore, in normoxia, HIF $1 \alpha$ appears as a lower molecular weight than under hypoxic conditions. (b) Immunoprecipitation of HIF1 $\alpha$ from hypoxic U2OS, HEK293 or HeLa lysates followed by phosphatase treatment resulted in a decrease in molecular weight as observed in (a), demonstrating the different molecular weights of HIF $1 \alpha$ under different oxygen tensions are due to phosphorylation. 
a
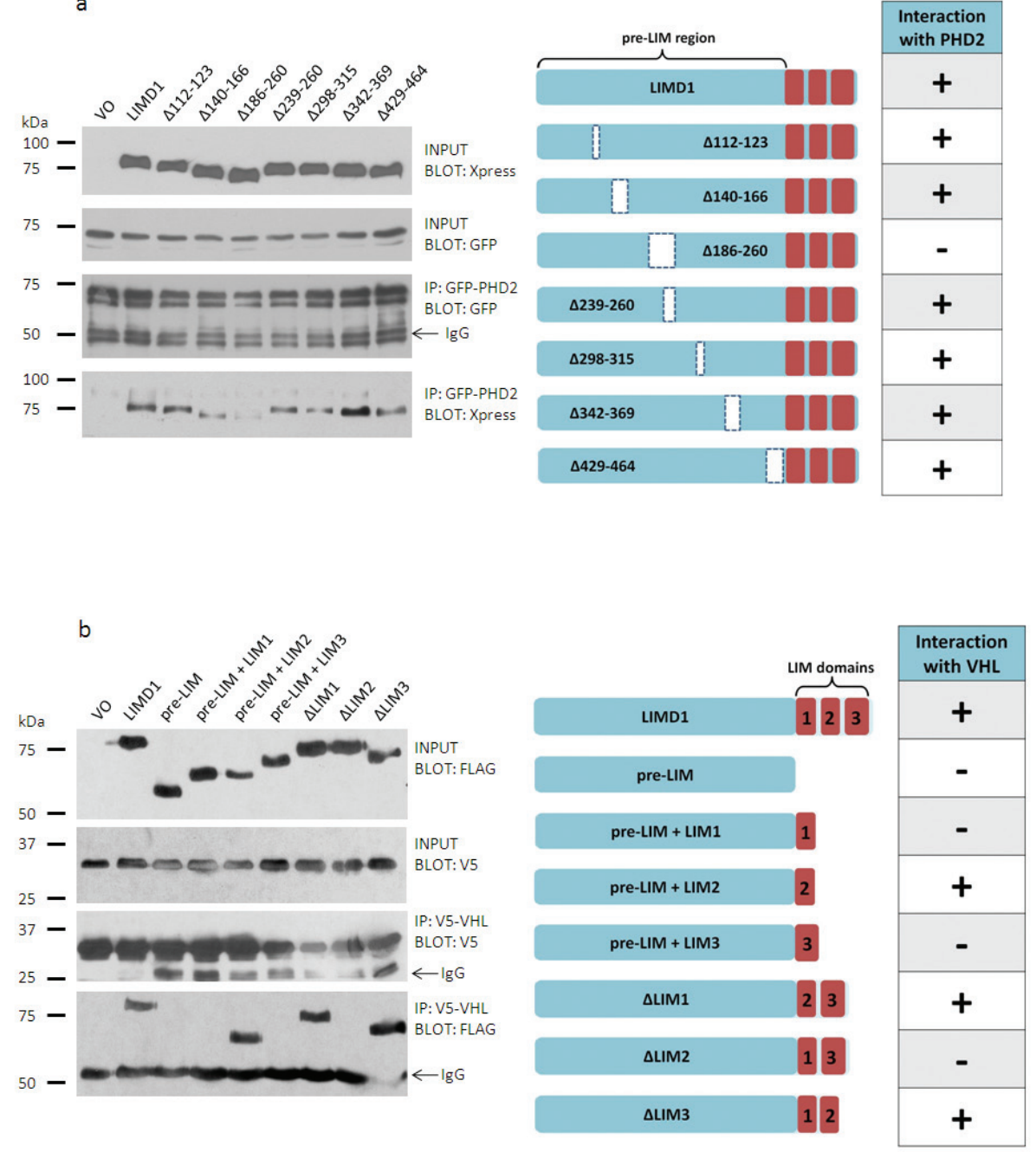

Figure S4 Mapping of the PHD2 and VHL binding domains within LIMD1. Xpress vector only, wild type LIMD1 or indicated LIMD1 deletion mutants were co-transfected with (a) GFP-PHD2 or (b) V5-VHL into U2OS cells. PHD2 or VHL were immunoprecipitated with GFP or
V5 antibodies and binding of LIMD1 and mutants to PHD2/VHL was assayed by Western blot. PHD2 binds to within amino acids $186-260$ of LIMD1 ( $\triangle 186-260$ ), whereas VHL binds to the second LIM domain ( $\triangle$ LIM2). 


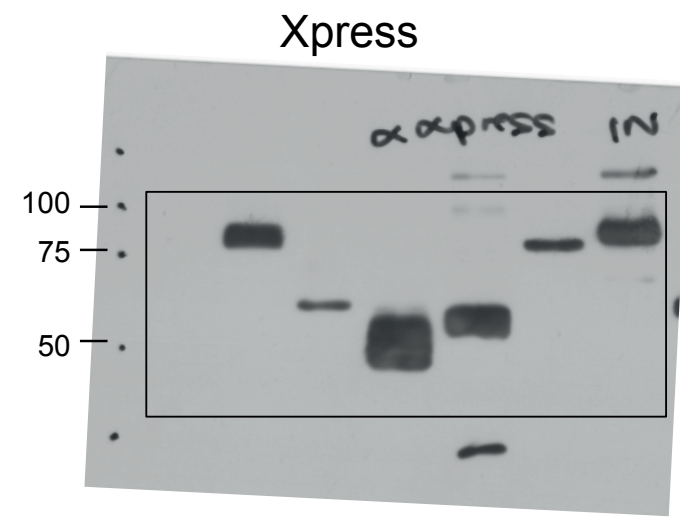

Fig 1a right panel

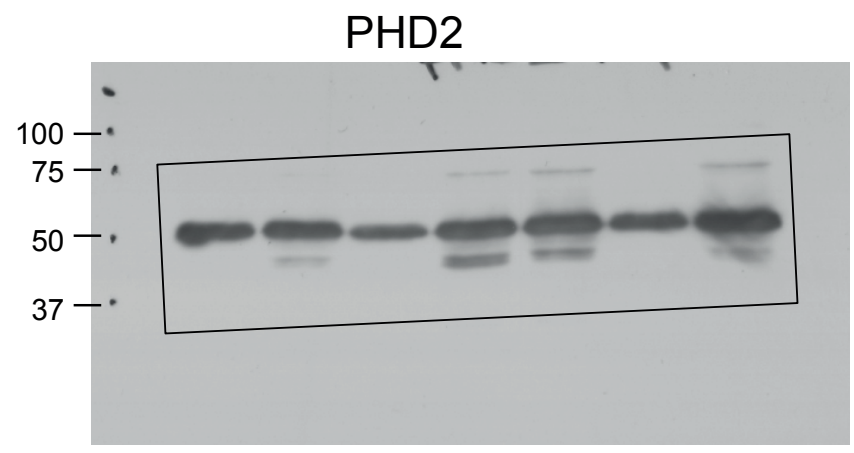

Fig 1a right panel

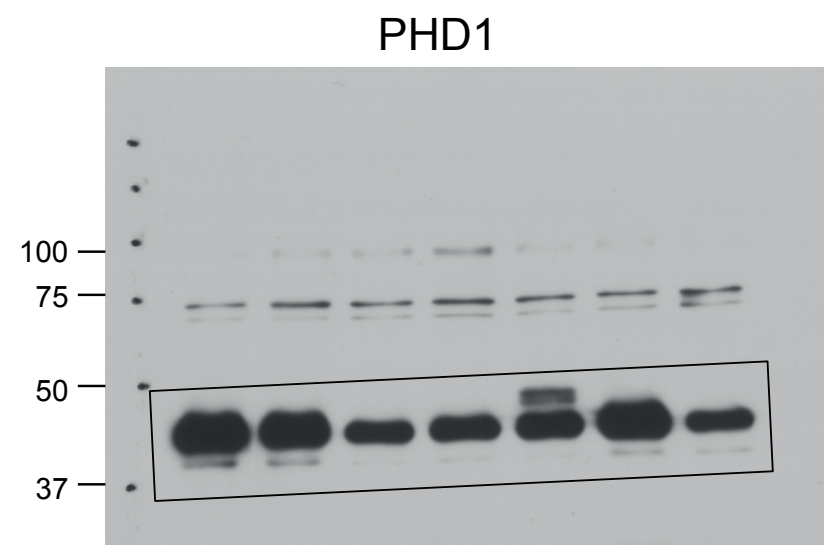

Fig 1a right panel

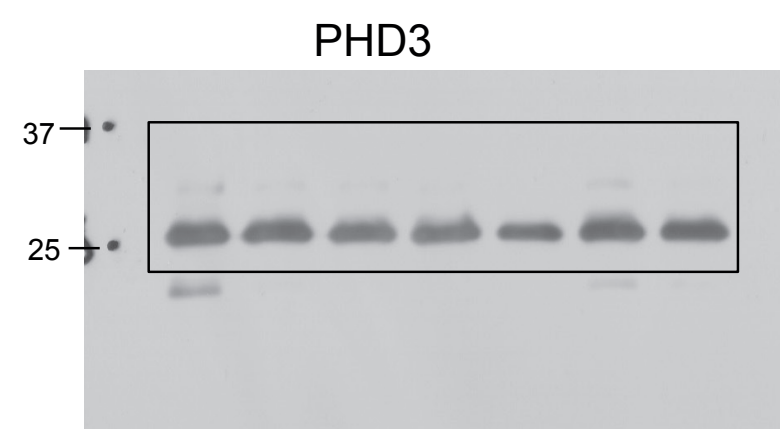

Fig 1a right panel

Figure S5 Full scans 
Xpress

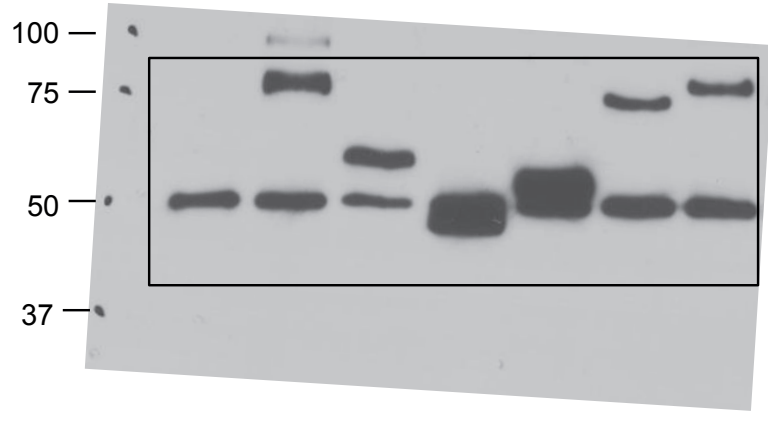

Fig 1a left panel

PHD2

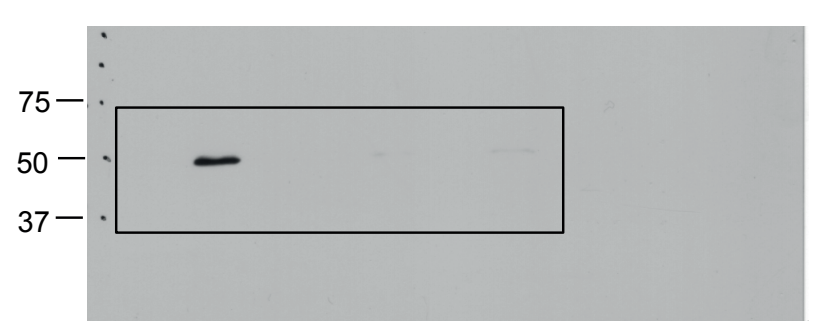

Fig 1a left panel

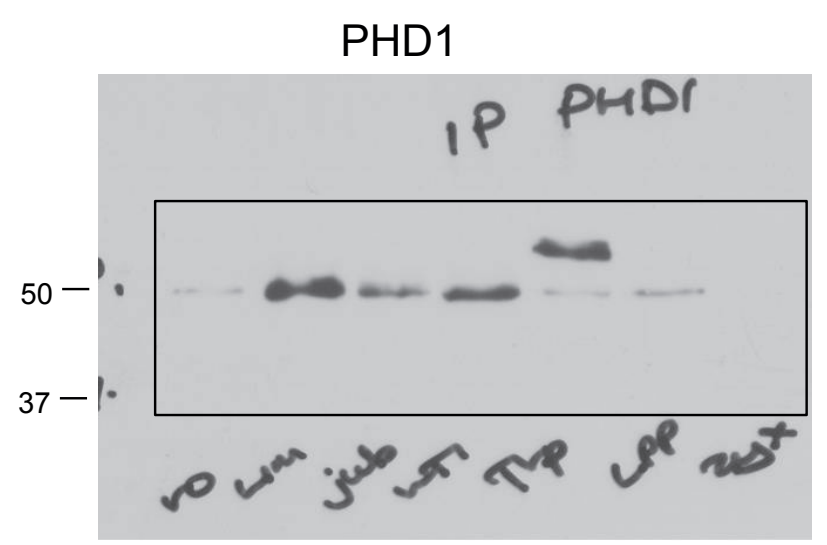

Fig 1a left panel

PHD3

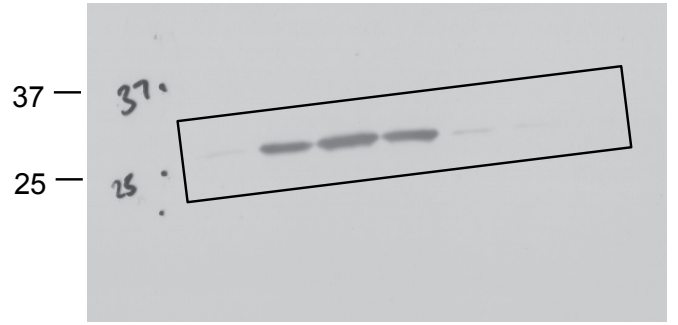

Fig 1a left panel 


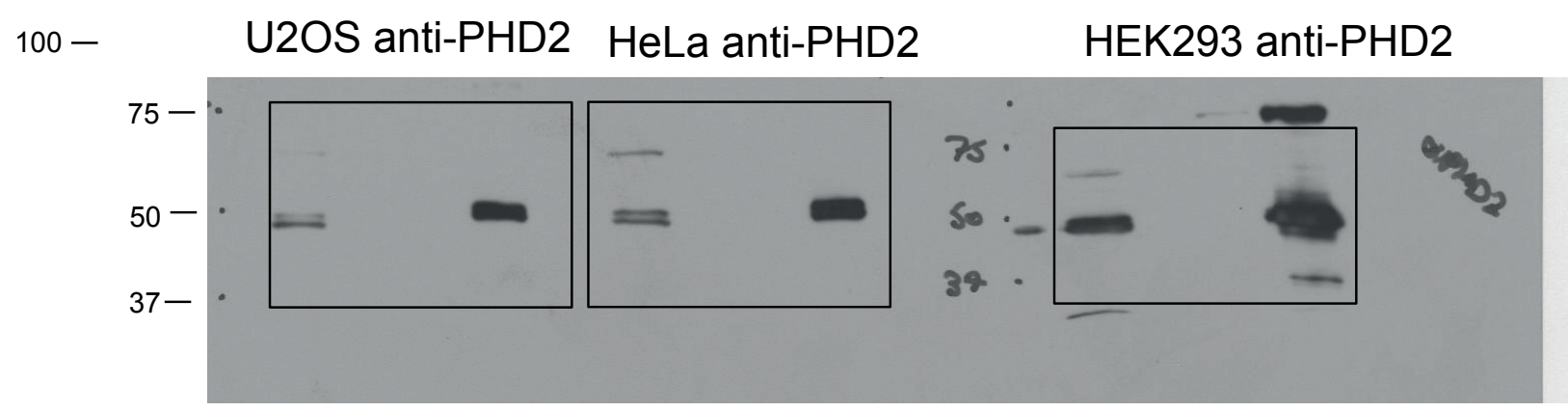

Fig. 1b

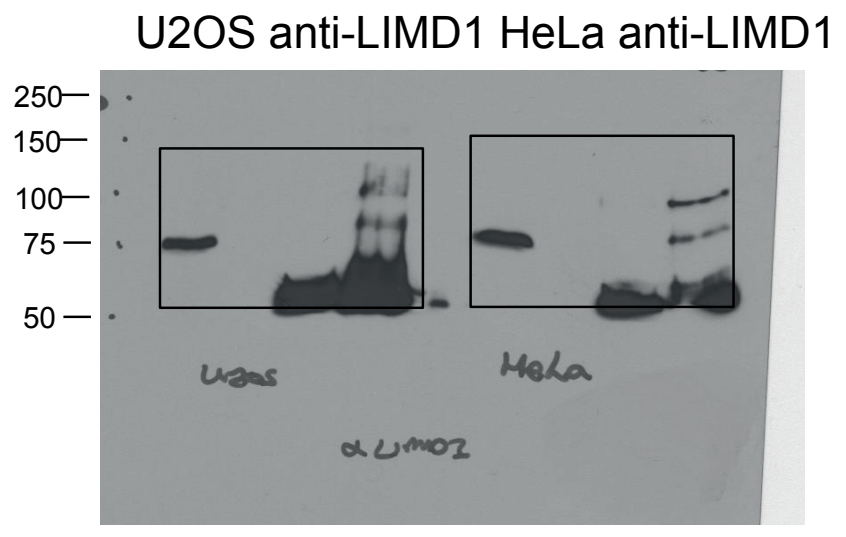

Fig. 1b

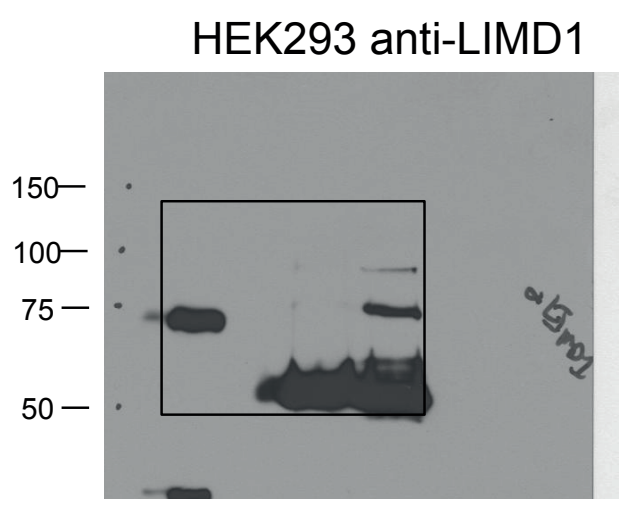

Fig. 1b 


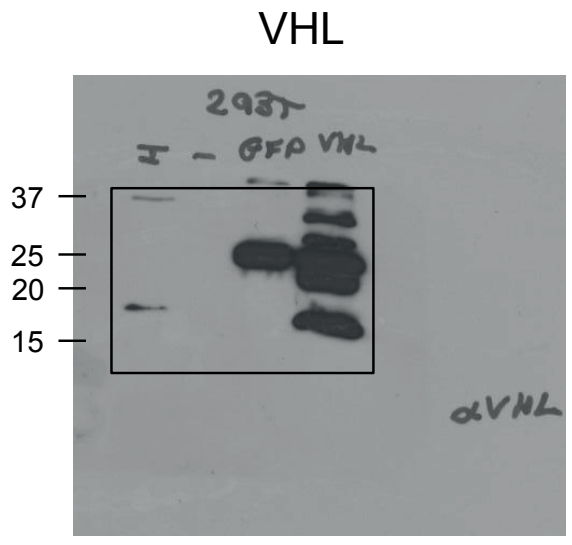

Fig. 1c

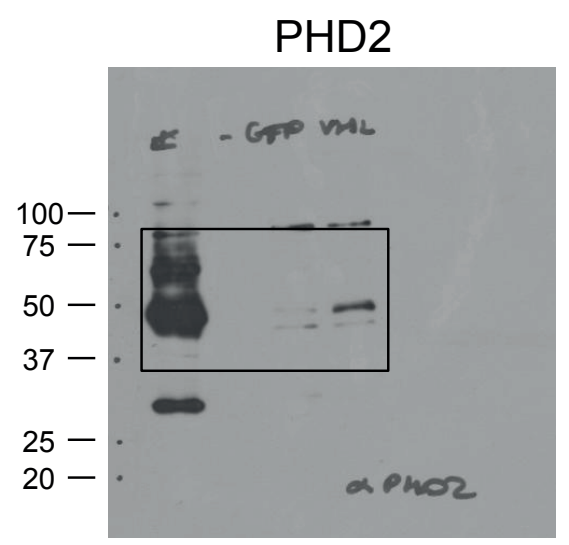

Fig. 1c

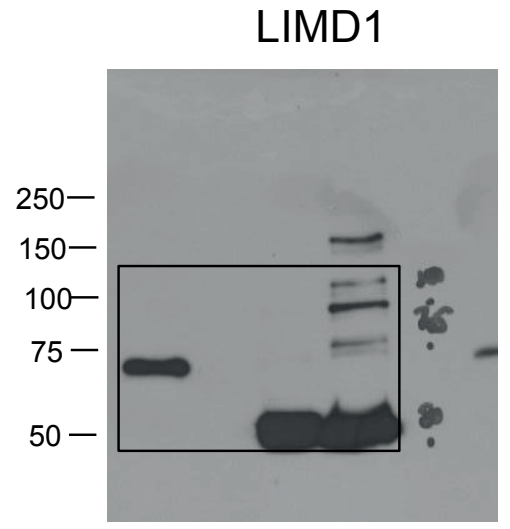

Fig. 1c

\section{ElonginB}

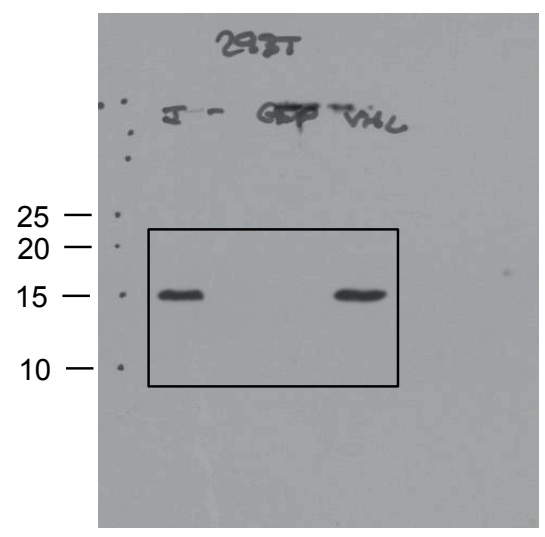

Fig. 1c

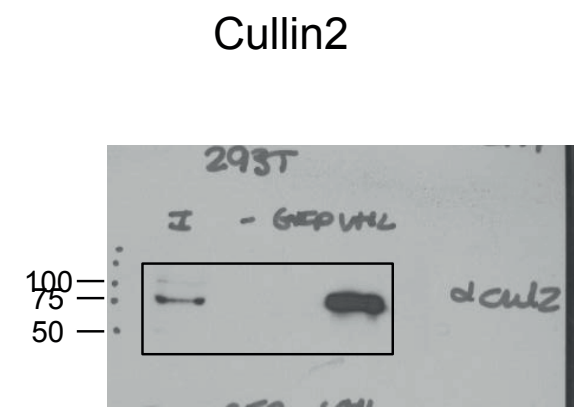

Fig. 1c 


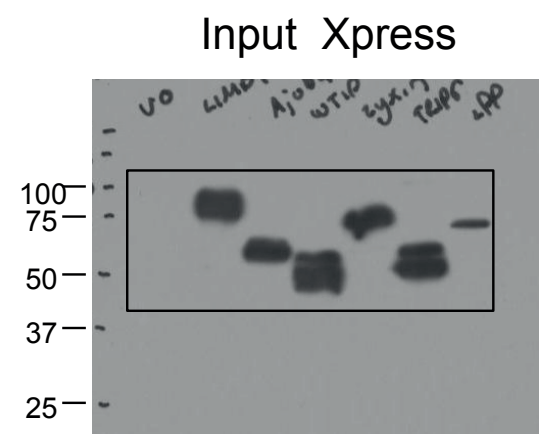

Fig. 1d

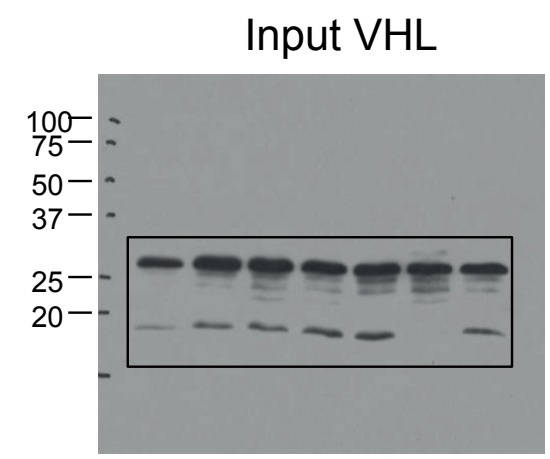

Fig. 1d

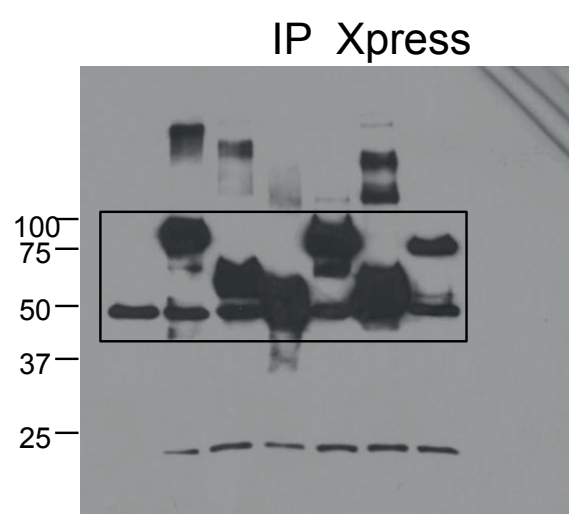

Fig. 1d
IP Xpress Blot VHL

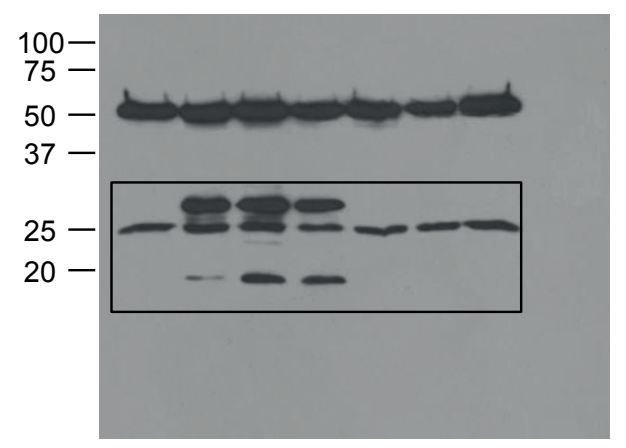

Fig. 1d

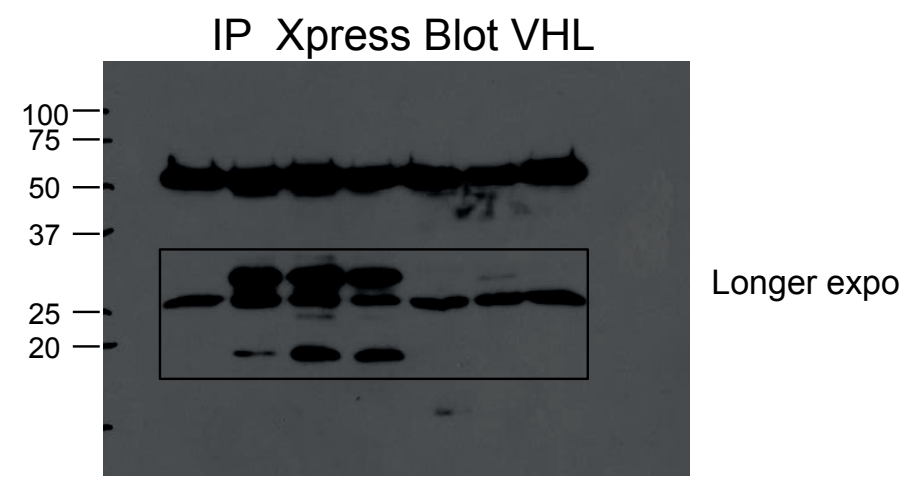

Fig. 1d 


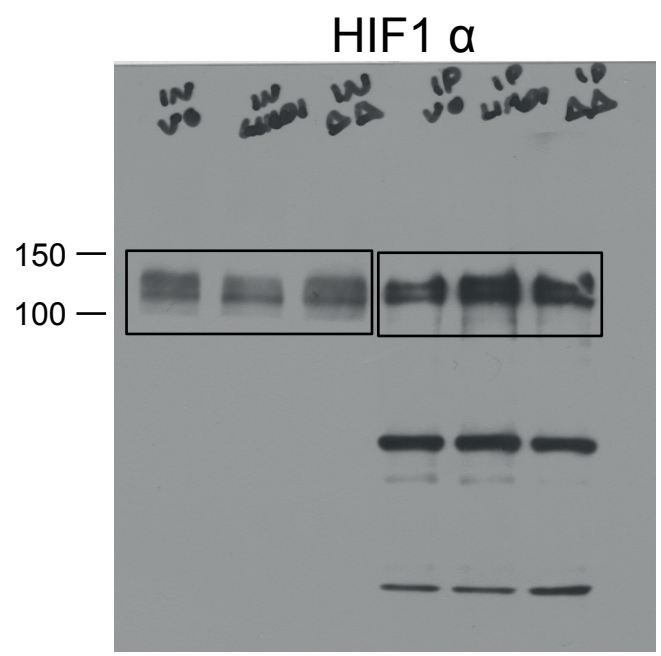

Fig. 1e

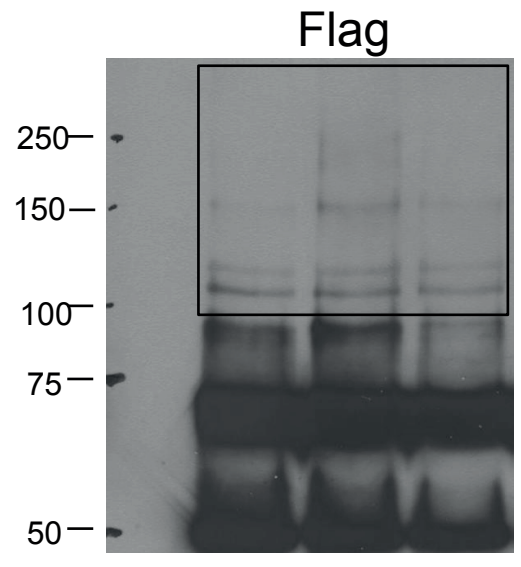

Fig. 1e

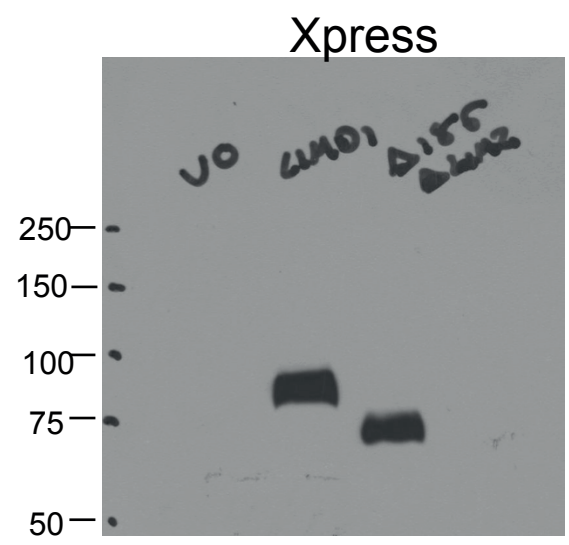

Fig. 1e

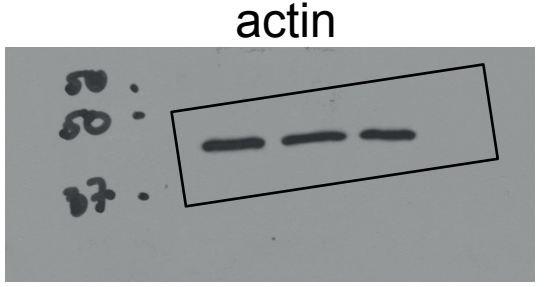

Fig. 1e 


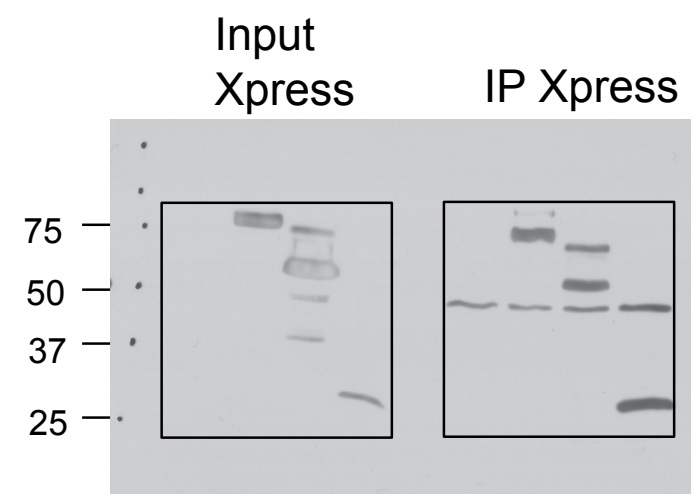

Fig. 1g

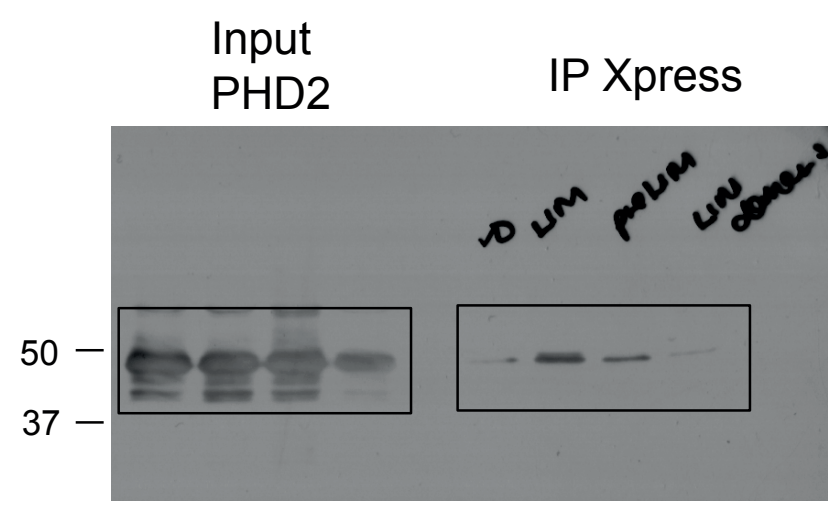

Fig. $1 \mathrm{~g}$

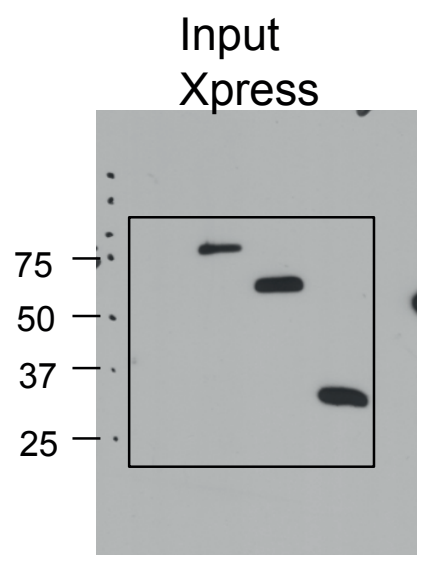

Fig. 1h

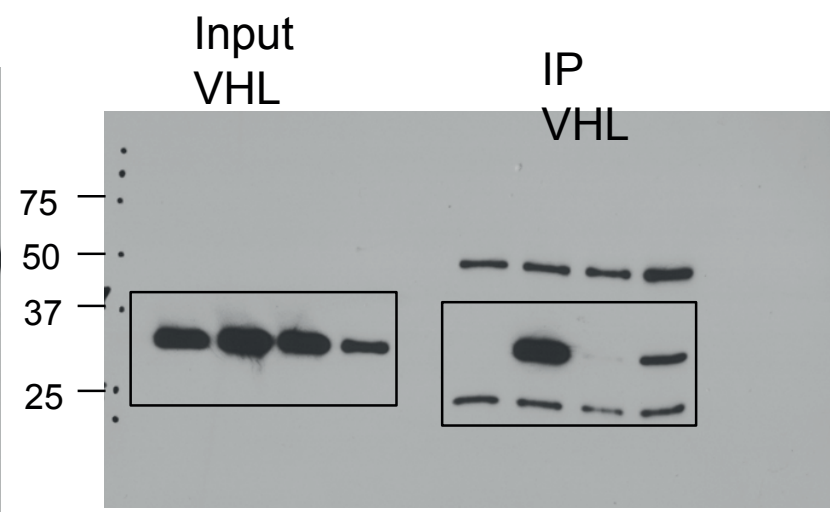

Fig. 1h

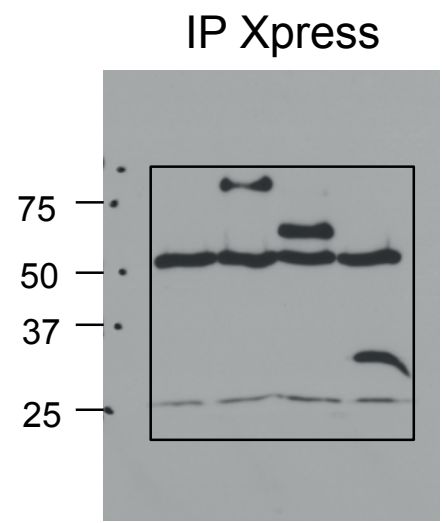

Fig. 1h 


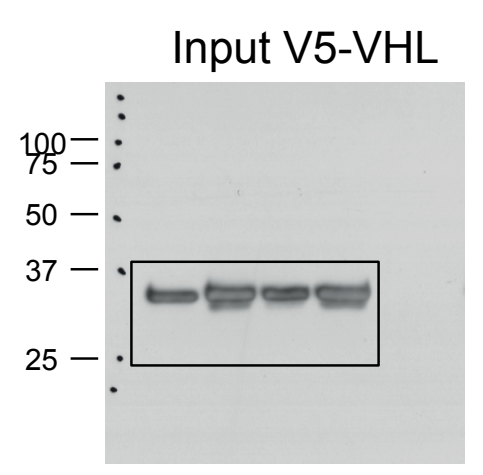

Fig. 1i

IP V5 Blot V5

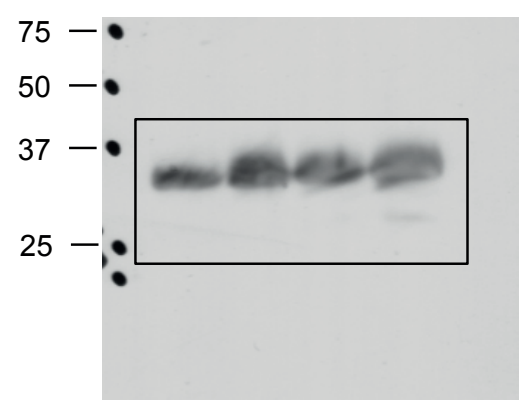

Fig. $1 \mathrm{i}$

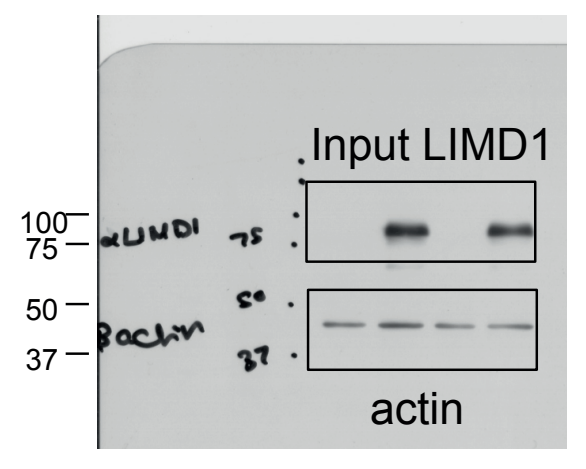

Fig. 1i

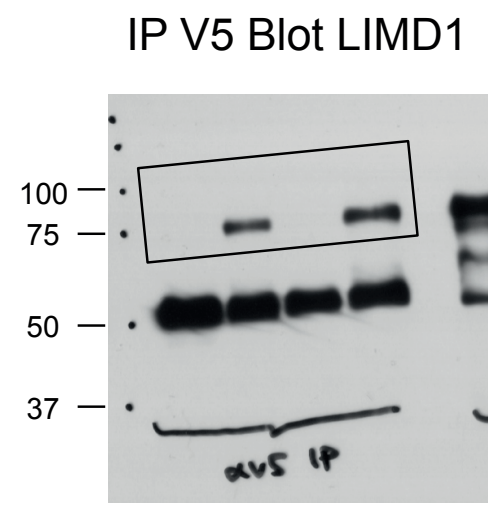

Fig. 1i

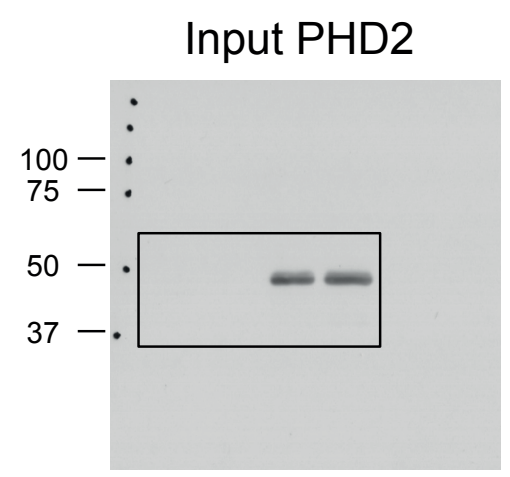

Fig. 1i

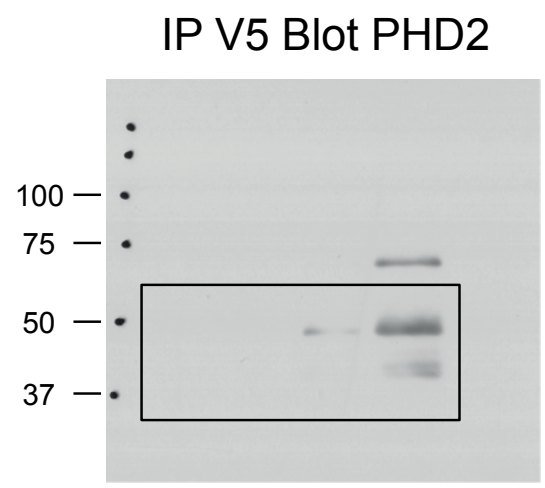

Fig. $1 \mathrm{i}$ 


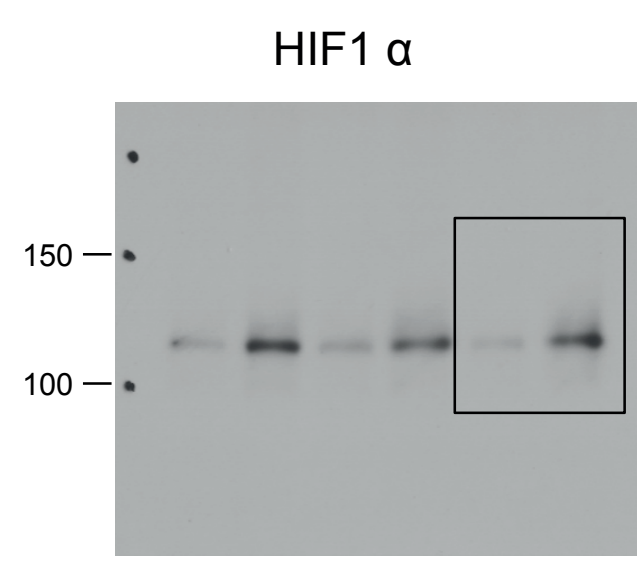

Fig. 2d

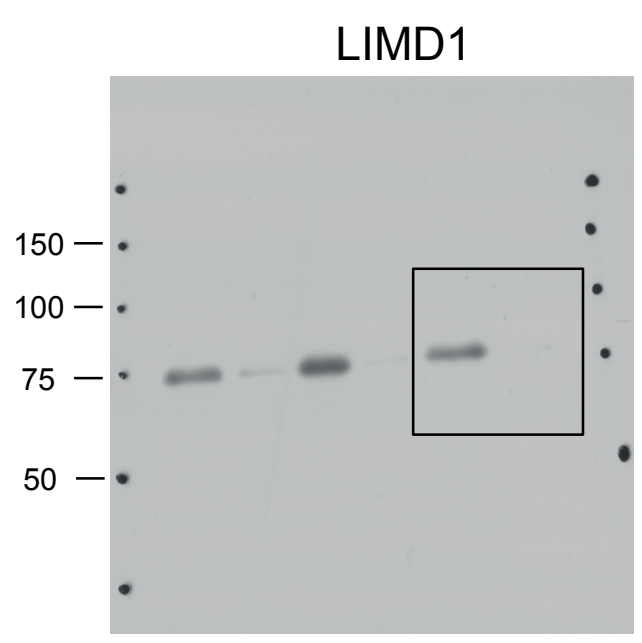

Fig. 2d

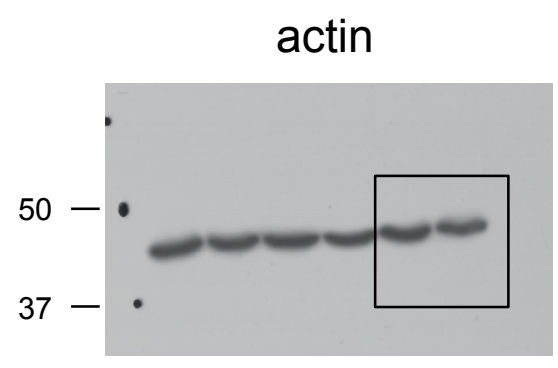

Fig. 2d 
HIF1 a
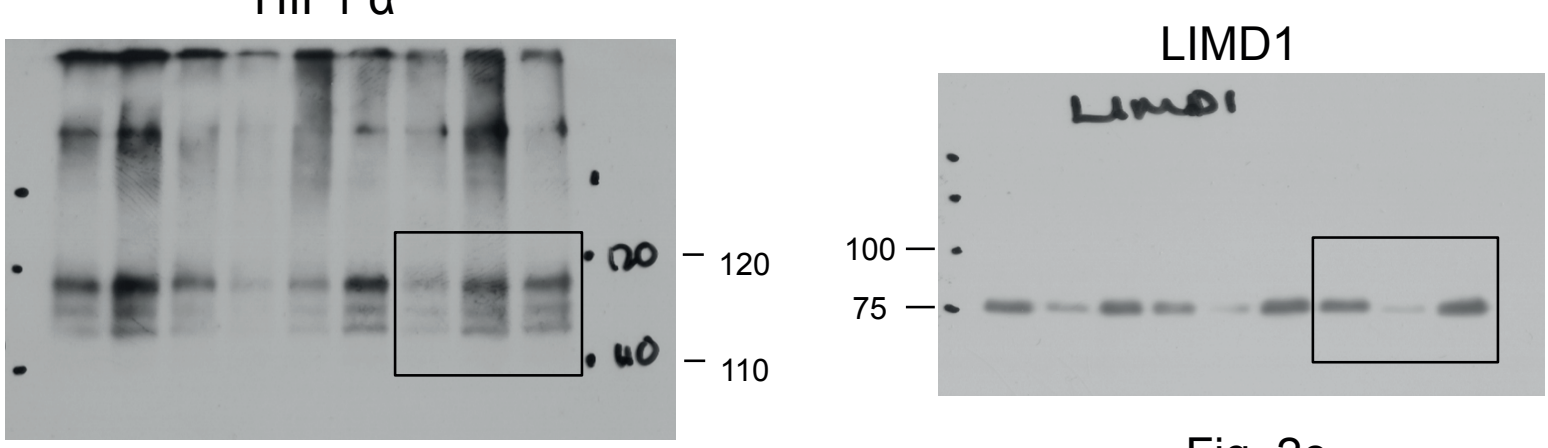

Fig. $2 \mathrm{e}$

Fig. $2 e$
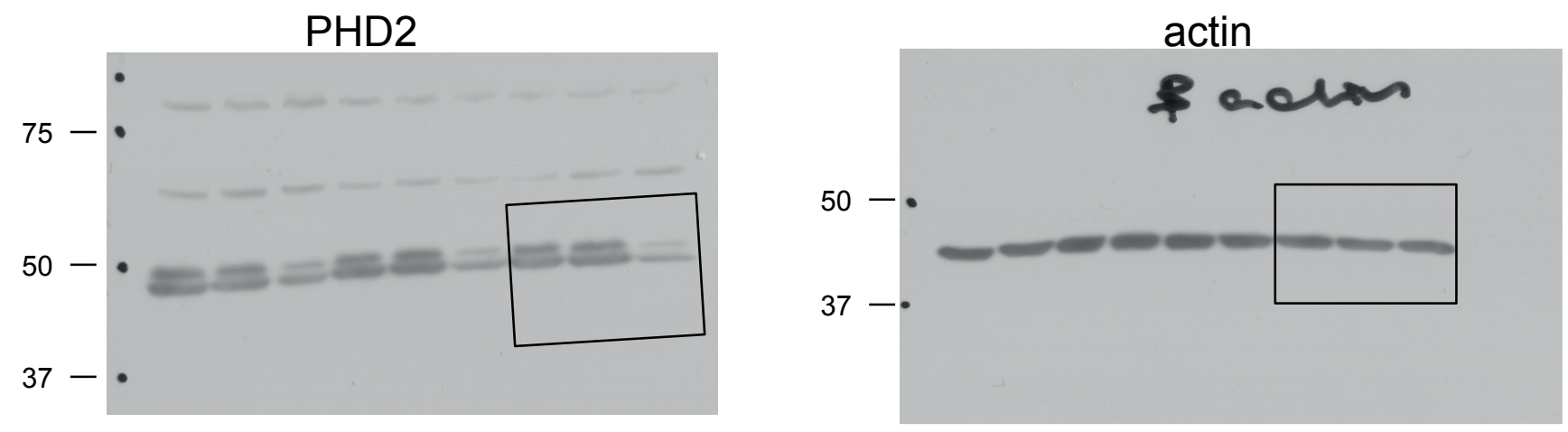

Fig. 2e

Fig. $2 \mathrm{e}$ 


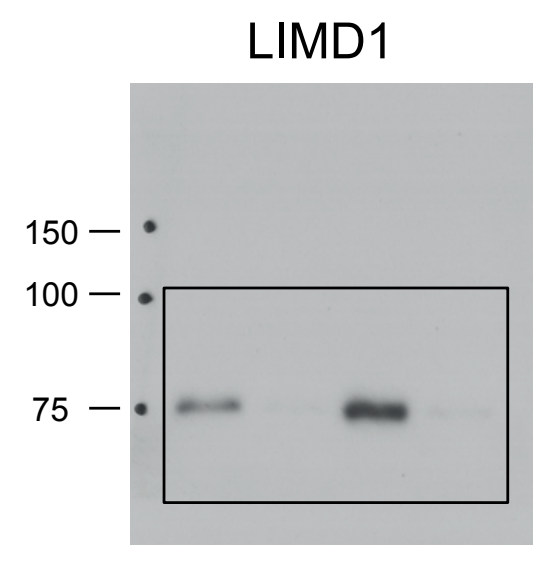

Fig. $2 f$

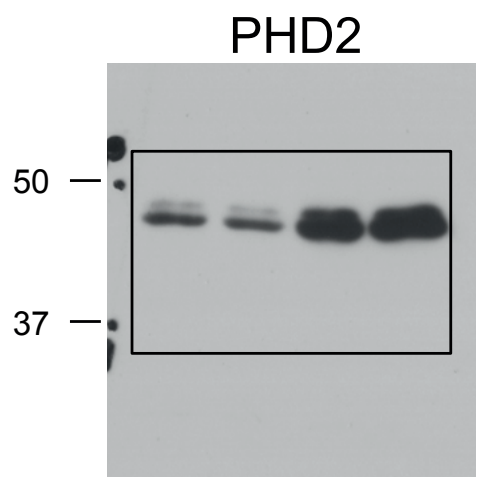

Fig. $2 f$

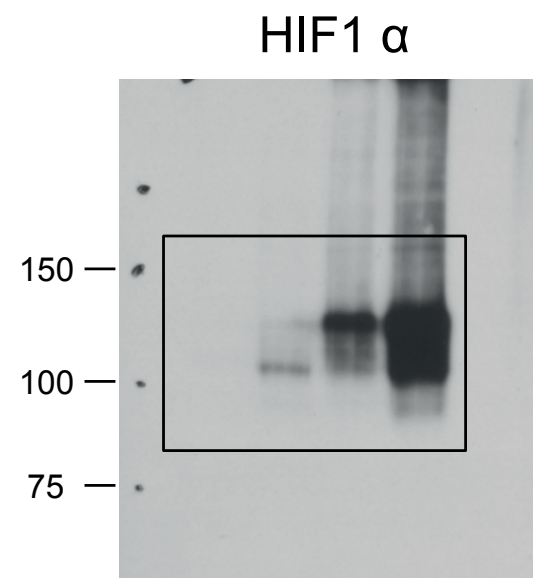

Fig. $2 f$

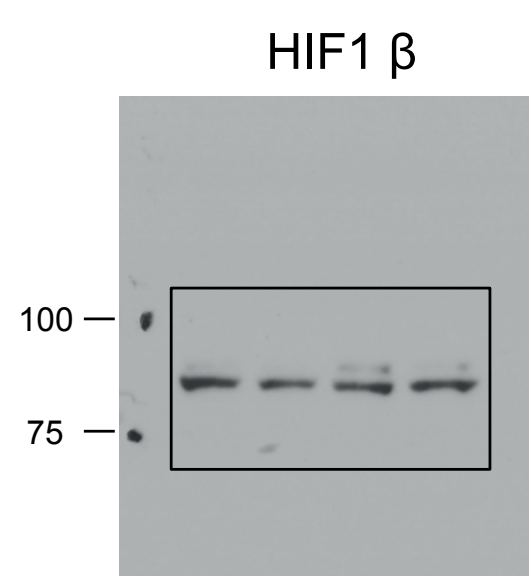

Fig. $2 f$

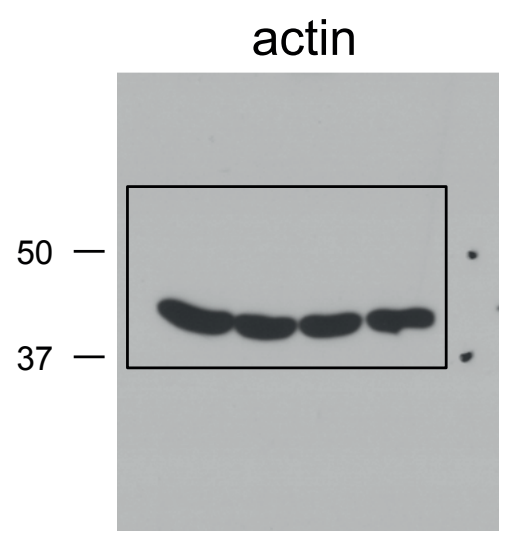

Fig. $2 f$ 


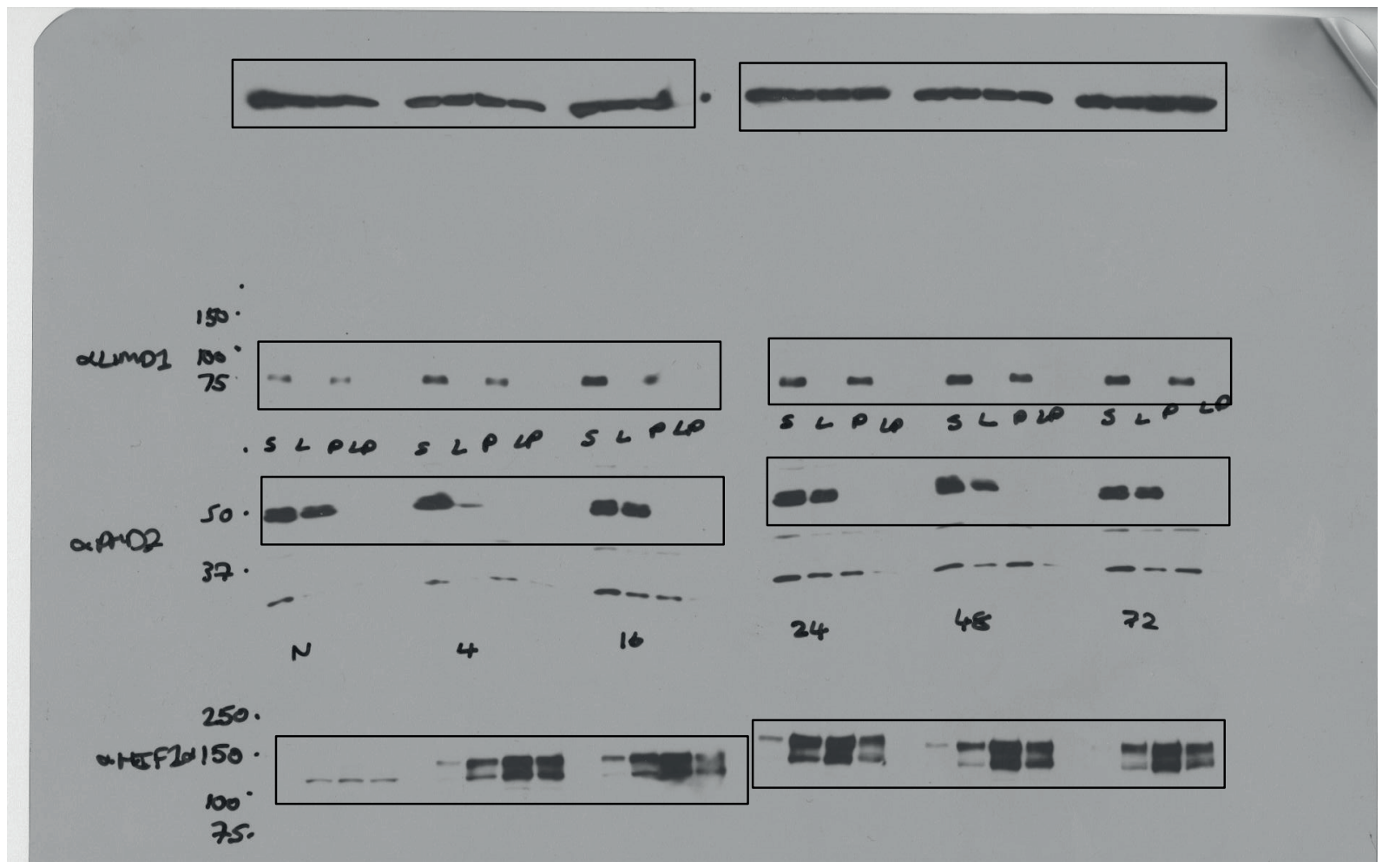

actin

LIMD1

PHD2

HIF1 $\alpha$

(1min)

Fig. $2 g$ 


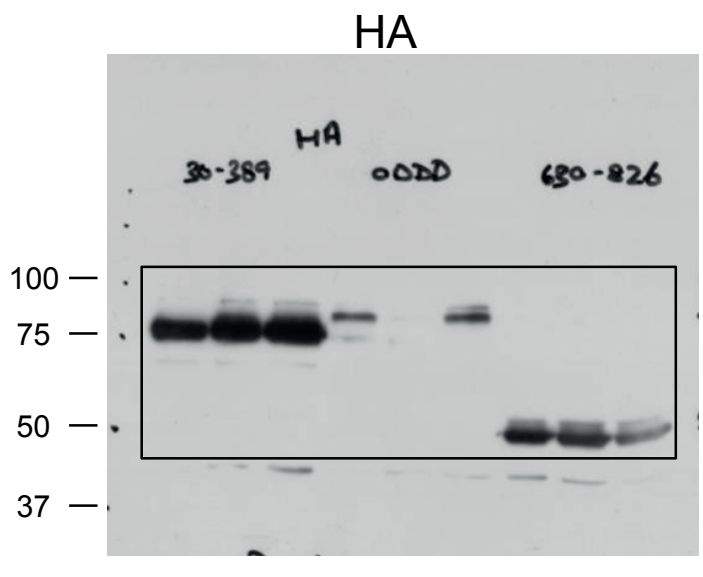

Fig. 3a

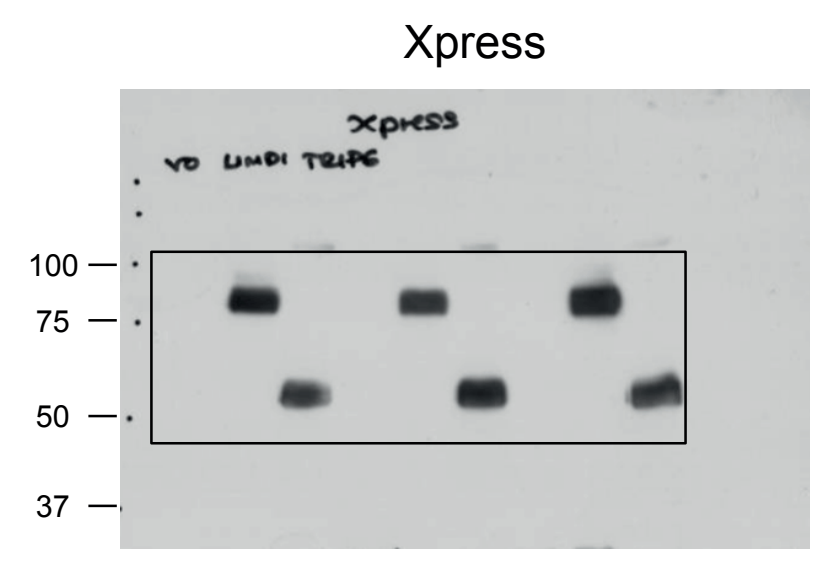

Fig. 3a

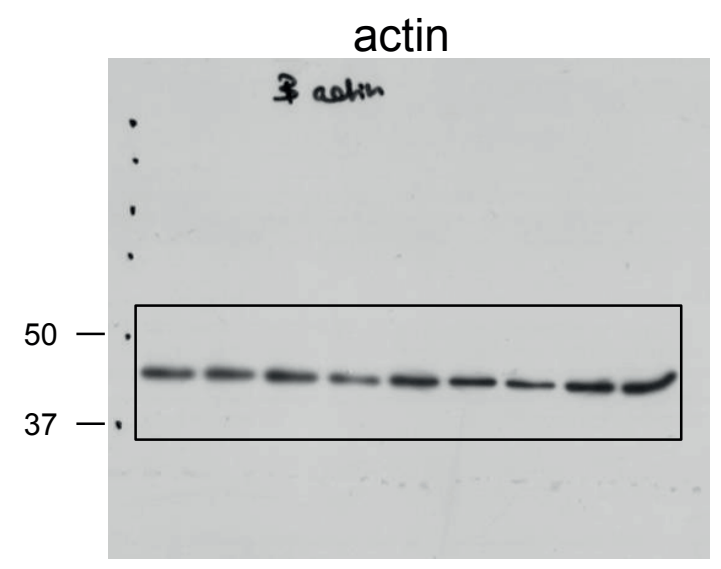

Fig. 3a 

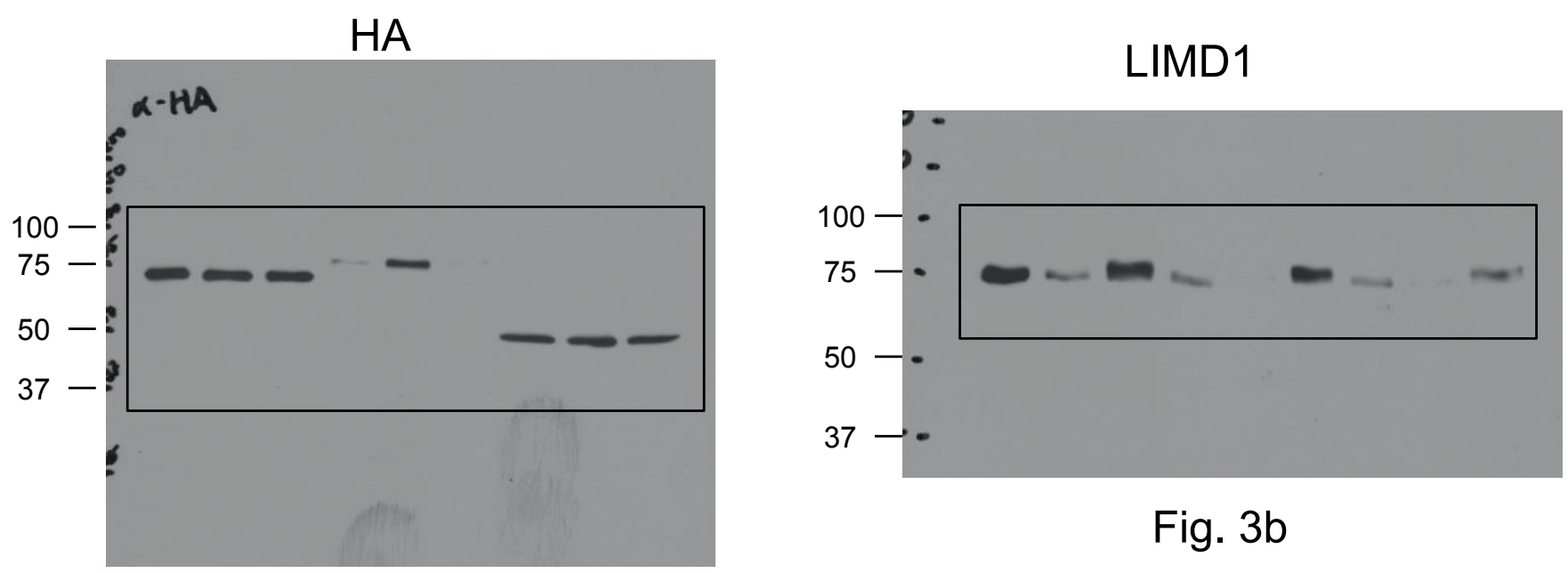

Fig. 3b

Fig. 3b

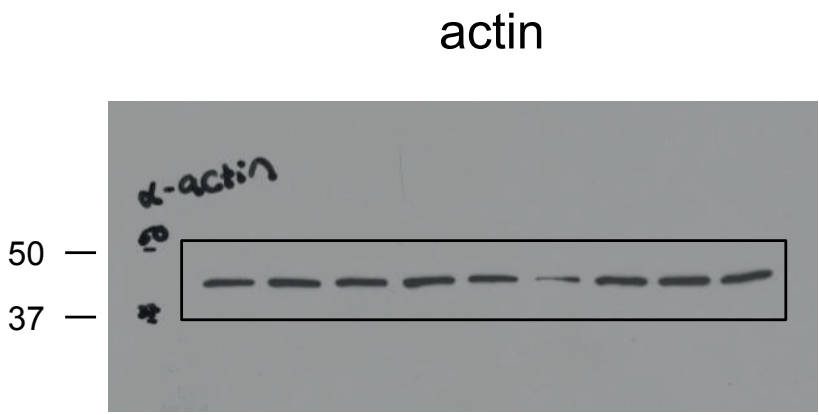

Fig. $3 b$ 


\section{Xpress}

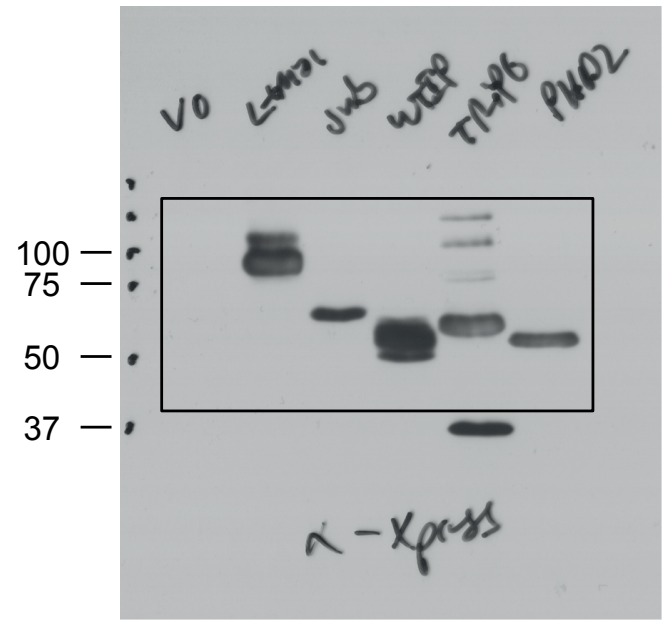

HA-ODD

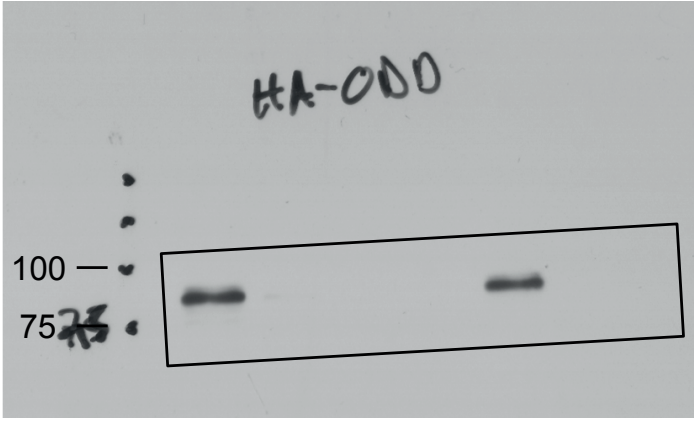

Fig. 3c

Fig. 3c

actin

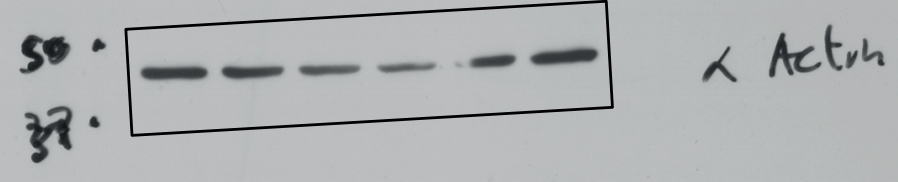

Fig. 3c 


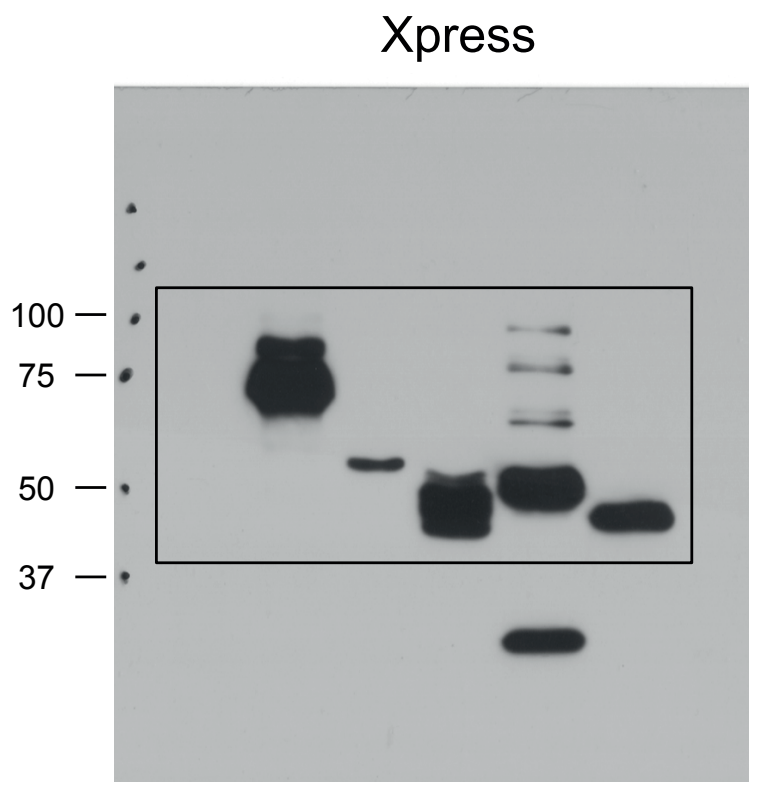

Fig. 3d

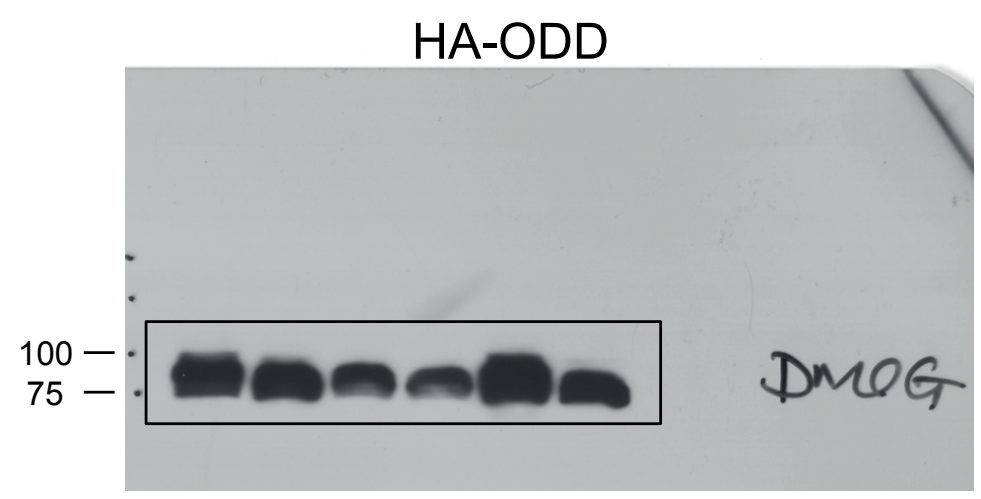

Fig. 3d

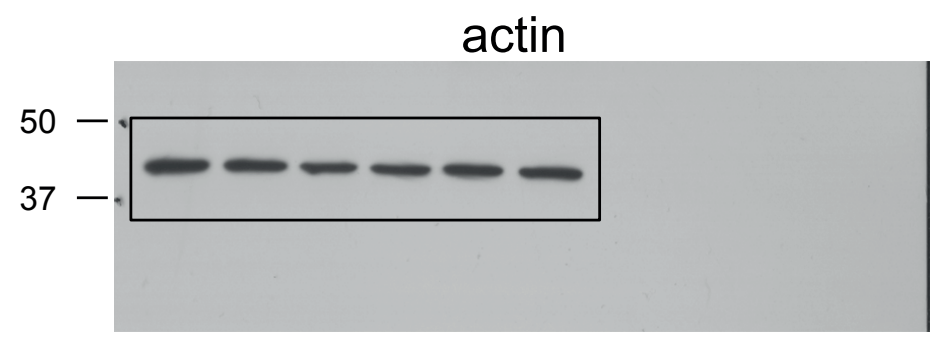

Fig. 3d 

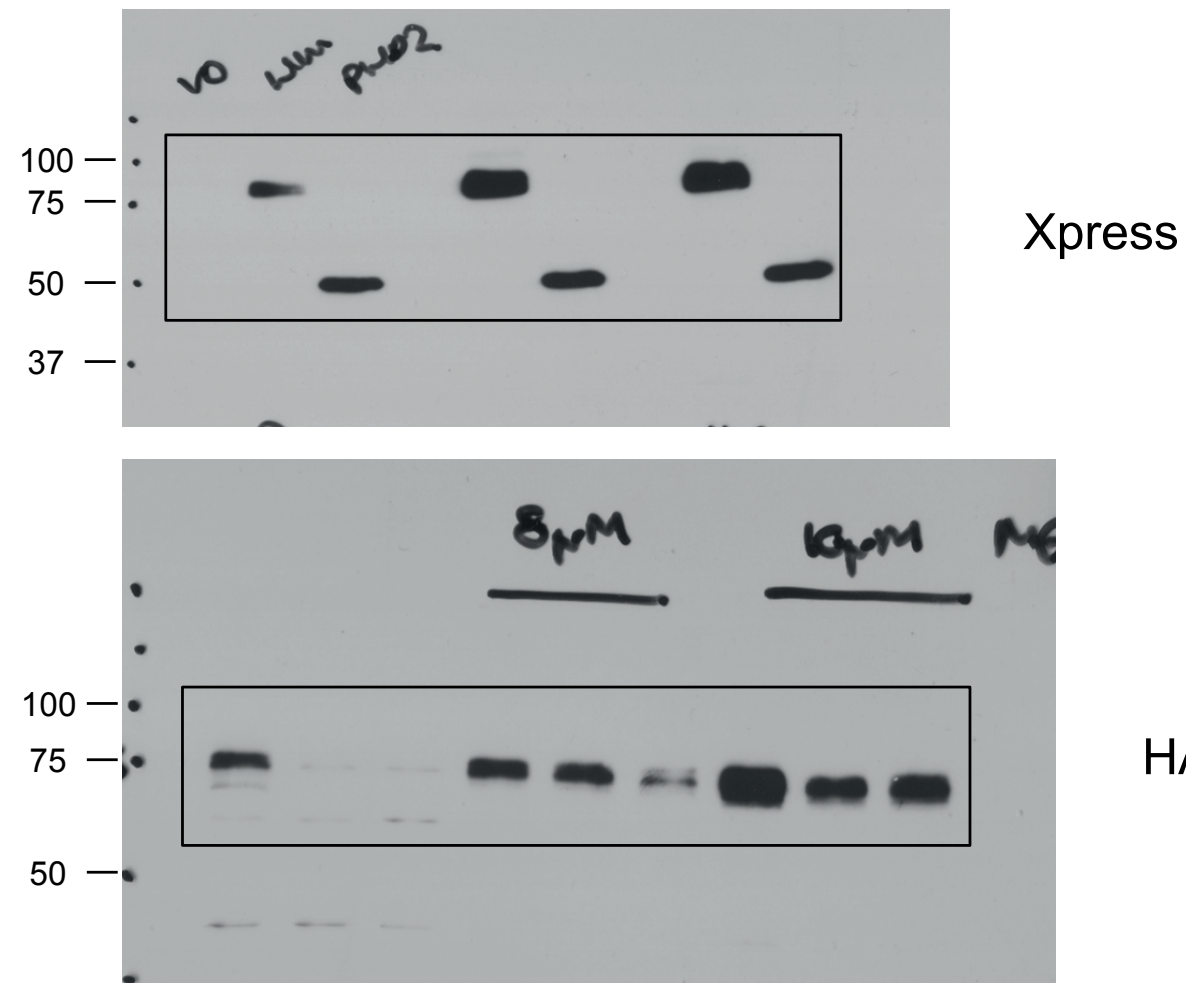

HA-ODD

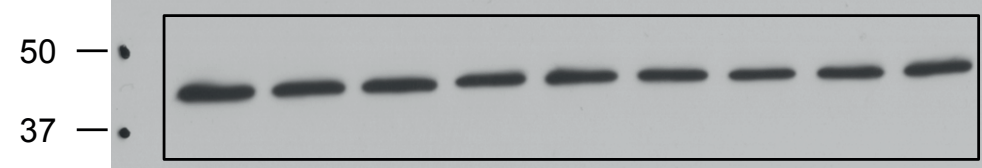

actin

Fig. 3e 


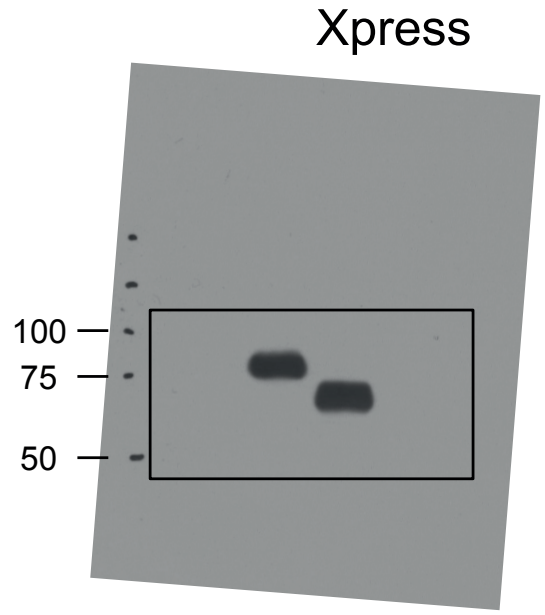

Fig. $3 f$

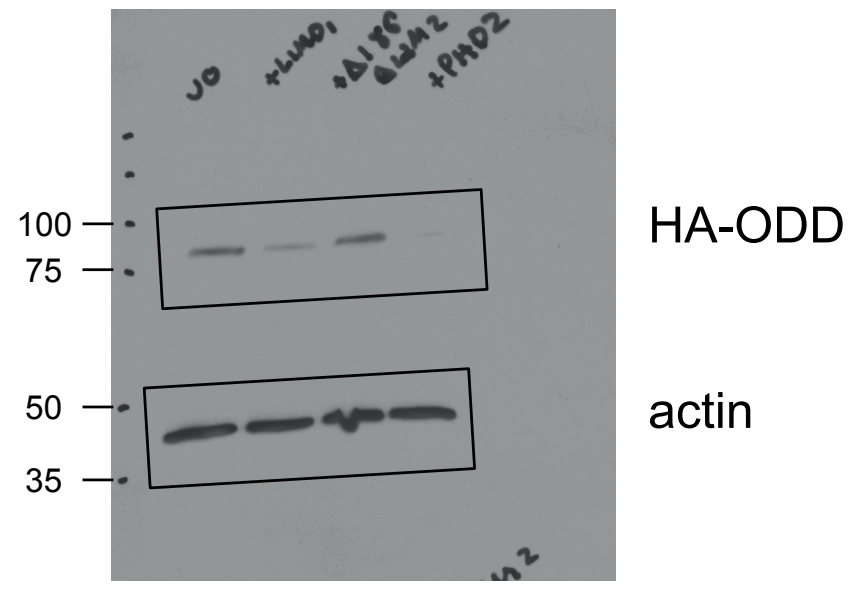

Fig. $3 f$ 


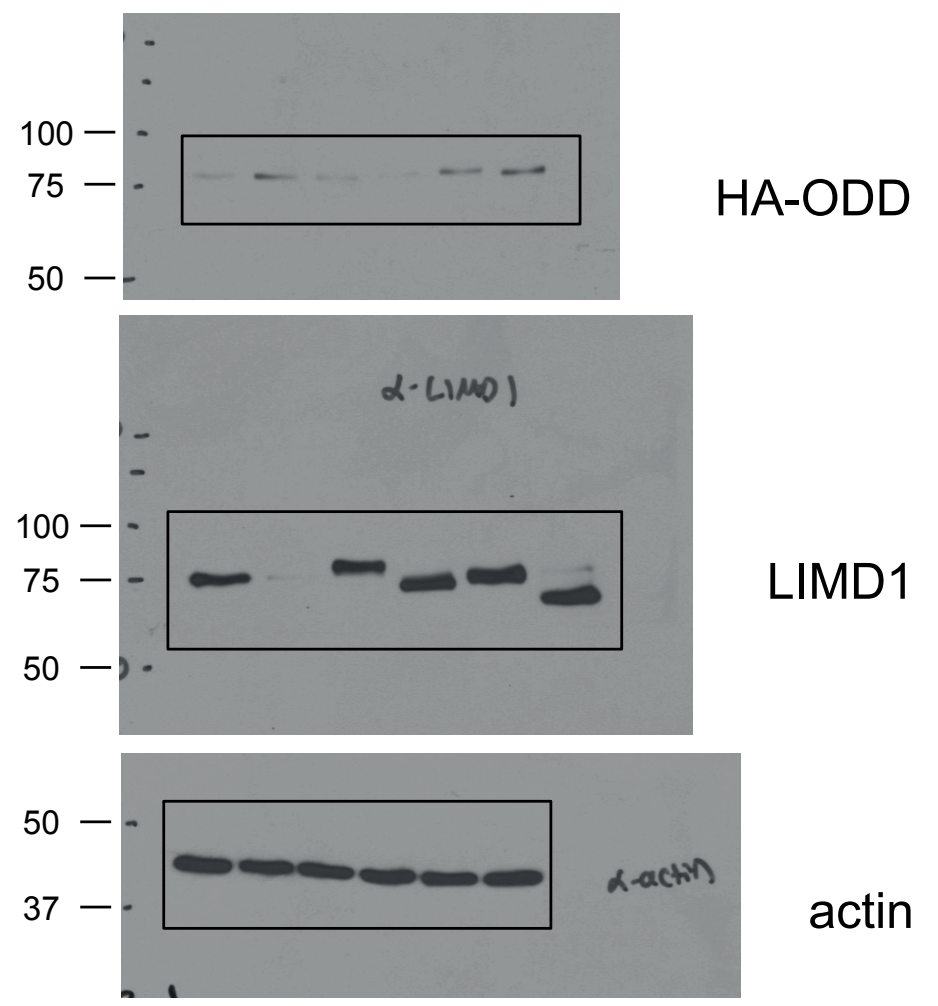

Fig. 3g 
SUPPLEMENTARY INFORMATION

Supplementary Tables

Table S1 List of all Primary antibodies use in this study together with the indicated product information.

Table S2 List of all Secondary antibodies use in this study together with the indicated product information.

Table S3 qRT-PCR Primer List for indicated human genes 